\title{
Optimal Long Term Investment in a Jump Diffusion Setting: A Large Deviation Approach
}

$\mathrm{Ba}$ Chu 


\title{
Optimal Investment and Asymmetric Risk for A Large Portfolio: A Large Deviations Approach
}

\author{
Ba M Chu \\ Birkbeck College
}

\author{
John Knight \\ University of Western Ontario
}

3rd September 2005

\begin{abstract}
In this study, we propose a new method based on the large deviations theory to select an optimal investment for a large portfolio such that the risk, which is defined as the probability that the portfolio return underperforms an investable benchmark, is minimal. As a particular case, we examine the effect of two types of asymmetric dependence; 1) asymmetry in a portfolio return distribution, and 2) asymmetric dependence between asset returns, on the optimal portfolio invested in two risky assets. Furthermore, since our analysis is based on a parametric framework, this allows us to formulate a close-form relationship between the measures of correlation and the optimal portfolio. Finally, we calibrate our method with equity data, namely S\&P 500 and Bangkok SET. The empirical evidences confirm that there is a significant impact of asymmetric dependence on optimal portfolio and risk.
\end{abstract}

JEL classification: C4; D8; G11

Keywords: Optimal portfolio; The Edgeworth expansion; Asymmetric Risk; Large deviations; Asymmetric Gamma distribution; Nonlinear correlations; Value at Risk

${ }^{*}$ Corresponding author: Stephen Satchell, Trinity College, Cambridge CB2 1TQ, the United Kingdom; E-mail: ses11@econ.cam.ac.uk 


\section{INTRODUCTION}

The main purpose of this study is to develop the idea, that is proposed in Stutzen (2003), Stutzer (2001a), Stutze (2004), Stutze (2000) and Stutzen (2001b), of using the large deviations theory in the problem of optimal portfolio selection. That is, an investor who is averse to losing more than an investable benchmark wants to choose an optimal portfolio that maximizes an expected utility function. Thus, both the criterion of minimization of the likelihood that the portfolio return falls less than a benchmark return and the criterion of maximization of an expected utility function can be reconciled.

Value at Risk (VaR) is a measure of market risk of a particular portfolio; it basically shows the maximum loss over a period at a given confidence level. Many new approaches have been developed. Popular approaches include historical simulation techniques; techniques based on parametric models such as Wong and So (2004), and methods on semiparametric estimations as proposed by Fan and Gu (2003). Alternatively, the underperformance probability (see Stutze, 2003) can be used as a measure to gauge risk; it shows the likelihood that the portfolio return underperforms a given benchmark return. Hereby, the first purpose of this paper is to apply the large deviations theory to estimate the optimal portfolio and the underperformance probability (i.e., asymmetric risk) for a large portfolio in view of two types of asymmetry in equity returns (Patton, 2004): 1) asymmetry in return distributions 2) asymmetric dependence between two or more equity returns. We also propose a general approach to choose optimal investment in a large number of assets. Although this method does not require the measures of correlation to be estimated, those measures or their connections can be recovered as functions of the optimal portfolio. Furthermore, we provide some large deviations theorems that state the asymptotic relationship between asymmetric risk and expected utility. In particular, by imposing the asymmetric Gamma distribution for the portfolio return, we can investigate the special case that there are two risky assets. The Edgeworth expansion is used to examine the effect of skewnesses and linear/nonlinear correlations on optimal portfolio and its asymmetric risk or risk bound, whereby a formal test can be formulated. 
The second part of this paper first provides a brief review of portfolio construction results under nonnormality and gives an elementary illustration of the large deviations principle that is used to construct the asymptotic estimate of the upper tail or the lower tail of a portfolio return distribution. Next, we provide major results on optimal portfolio selection for a large number of assets and analysis of the effect of skewnesses and correlations.

The third part is devoted to simulation and empirical evidences. Equity data of Bangkok SET index and S\&P 500 index are used to confirm that there is an effect of skewnesses and nonlinear correlations on optimal portfolio and asymmetric risk; and the optimal portfolio depends on investor's attitude toward risk.

The final part is an appendix containing proofs of the large deviations theorems given in the main text.

\section{OPTIMAL PORTFOLIO AND ASYMMETRIC RISK}

\subsection{Minimization of the Underperformance Probability}

The method of optimal investment selection originates from Markowit (1952) who defined optimal portfolio as the proportion that maximizes the mean of a portfolio return for a given level of risk as gauged by the standard deviation. Basically, this model assumes that the portfolio return is normal distributed so that high moments such as skewness and kurtosis that are prevalent in asset returns are disregarded (see e.g. Jondeau and Rockinge, 2003).

Another conventional procedure to construct an optimal portfolio is by using an expected utility. The basic idea of this method is to maximize a non-decreasing and concave expected utility function for an optimal investment strategy (see e.g. \ngersoll, [987). Choosing an appropriate functional form of investor's utility is problematic. Many utility functions such as the log utility, the power utility, and a class of HARA utility functions are proposed to capture investor's attitude to risk. Meanwhile, many authors such as Malevergne and Sornette (2002) propose that the optimal portfolio can be selected by maximizing an approximation of an expected utility function in terms of high order moments of wealth. However, since investors 
are often adverse to earning a portfolio return that is less than a benchmark return Stutzen, 2000), the rational investment criteria should involve minimization of the likelihood that the portfolio return underperforms a benchmark return. As Stutzer (2003) pointed out, the large deviations theory can be used to reconcile the criterion of maximizing an expected utility and that of minimizing the underperformance probability.

The underperformance probability that is sometimes interpreted as downside risk (see Menezes et al[, 1980) has the following properties:

- The underperformance probability is a special case of the lower partial moment CAPM that was first proposed by Bawa (1979), then extended by Harlow and Rad (1989). In this framework, the lower partial moment is defined as $L P M_{n}(r, P)=\int_{\infty}^{r}(r-\tilde{r})^{n} d P(\tilde{r})$. Hence, the underperformance probability is obtained when $n=0$. Baw (1978) argues that for an arbitrary probability distribution $P, L P M_{n}(r, P)$, that is calculated at a fixed boundary value $(r)$, appears to be a relevant scalar risk measure instead of the usual variance. This is due to the fact that there exists an intimate relationship between the Second Stochastic Dominance (SSD) and the lower partial moment (LPM). [See Theorem 1 in Bawa (1978).] Based on the generalized LPM, the LPM CAPM was constructed by Harlow and Rad (1989). They shows that the optimal market portfolios, that are not meanvariance efficient, may be SSD efficient because the SSD simply picks up the downside risk that is overlooked in the mean-variance framework; and uses those additional sources of risk in order to rationalize the mean-variance inefficiency of the optimal market portfolios. Since the SSD is based on the Von Neumann expected utility paradigm, the optimal portfolio, that minimizes the underperformance probability whilst maximizing a skewnesspreference expected utility is obviously SSD efficient.

- The underperformance probability has many advantages over the methods of using higher moments as measures of fluctuation. As Malevergne and Sornette 2002) pointed out, using high order cumulants as the risk measures would result in irrational behavior of an agent

\footnotetext{
${ }^{1}$ The SSD is defined on a set of utility functions $U$ such that $U^{\prime \prime \prime}>0, U^{\prime \prime}<0$, and $U^{\prime}>0$. Kand (1982) shows that these types of utility function support skewness preference.
} 
who tries to minimize risk. For instance, the fourth order cumulant $C_{4}=\mu_{4}-3 \mu_{2}^{2}$, where $\mu_{4}$ and $\mu_{2}$ are the fourth order central moment and the variance respectively, depicts an agent who tries to avoid the large risks whilst ready to accept the smallest ones. Therefore, the lower tail of a return distribution instead of its high order moments should be used as a coherent measure of risk.

Let $\tilde{r}_{i, t}$ for $i=1, . ., n$ and $t=1, . ., T$ denotes the arithmetic asset return as given by

$$
\tilde{r}_{i, t}=\frac{P_{i, t}-P_{i, t-1}}{P_{i, t-1}}
$$

Throughout this paper, $\tilde{r}_{i, t}$ are assumed IID, thus the probability that the one period portfolio return $R_{p, n}^{(\boldsymbol{\alpha})}(\omega)=\sum_{i=1}^{n} \alpha_{i} \tilde{r}_{i}$, where $\boldsymbol{\alpha}=\left\{\alpha_{1}, . ., \alpha_{n}\right\}$ are investable proportions, exceeds a riskfree interest rate is

$$
P\left\{R_{p, n}^{(\boldsymbol{\alpha})}(\omega)>r\right\} \lesssim \exp \left\{-\max _{\substack{\theta>0 \\ \mathbf{1}^{\prime} \boldsymbol{\alpha}=1}}\left\{\theta r-\log E\left[\exp \left\{\theta R_{p, n}^{(\boldsymbol{\alpha})}(\omega)\right\}\right]\right\}\right\}
$$

(11) is first established by Cramer. The RHS of (四) is also known as Chernoff's bound. [See Dembo and Zeitoun (1999, page 26).] The intuition of (11) is that the upper bound can be made as close as possible to the upper tail of the return distribution by increasing the number of assets $(n)$. The duality between $P\left\{R_{p, n}^{(\boldsymbol{\alpha})}(\omega)>r\right\}$ and $P\left\{R_{p, n}^{(\boldsymbol{\alpha})}(\omega)<r\right\}$ can be seen in Figures i and ii.

Figure i shows that $\mathbf{1}_{[r, \infty)}\left(R_{p, n}^{(\boldsymbol{\alpha})}(\omega)\right) \leq \frac{\exp \left\{\theta R_{p, n}^{(\boldsymbol{\alpha})}(\omega)\right\}}{\exp \{\theta r\}}$ if $\theta>0$. Therefore, the upper tail probability can be written as

$$
\begin{aligned}
P\left\{R_{p, n}^{(\boldsymbol{\alpha})}(\omega)>r\right\}\left(=E\left[\mathbf{1}_{[r, \infty)}\left(R_{p, n}^{(\boldsymbol{\alpha})}(\omega)\right)\right]\right) & \leq E\left[\frac{\exp \left\{\theta R_{p, n}^{(\boldsymbol{\alpha})}(\omega)\right\}}{\exp \{\theta r\}}\right] \\
& =\exp \left\{-\left(\theta r-\log E\left[\exp \left\{\theta R_{p, n}^{(\boldsymbol{\alpha})}(\omega)\right\}\right]\right)\right\} \\
& =\exp \left\{-\Lambda_{n}(r, \theta, \boldsymbol{\alpha})\right\},
\end{aligned}
$$

where $\Lambda_{n}(r, \theta, \boldsymbol{\alpha})=\theta r-\log E\left[\exp \left\{\theta R_{p, n}^{(\boldsymbol{\alpha})}(\omega)\right\}\right]$.

Now, we can make the RHS of (2) as close as possible to the LHS by maximizing the deviations 
function $\Lambda(r, \theta, \boldsymbol{\alpha})$ w.r.t. $\theta$ and $\boldsymbol{\alpha}$, then sending $(n)$ to infinity.

Similarly, Figure ii shows that the lower tail probability is

$$
\begin{aligned}
P\left\{R_{p, n}^{(\boldsymbol{\alpha})}(\omega)<r\right\}\left(=E\left[\mathbf{1}_{(\infty, r]}\left(R_{p, n}^{(\boldsymbol{\alpha})}(\omega)\right)\right]\right) & \leq E\left[\frac{\exp \left\{\theta R_{p, n}^{(\boldsymbol{\alpha})}(\omega)\right\}}{\exp \{\theta r\}}\right] \\
& =\exp \left\{-\Lambda_{n}(r, \theta, \boldsymbol{\alpha})\right\}
\end{aligned}
$$

where $\theta<0$.

The RHS of (3) can be made as close as possible to the LHS by maximizing $\Lambda_{n}(r, \theta$, $\boldsymbol{\alpha})$ w.r.t. $\theta$ and $\boldsymbol{\alpha}$.

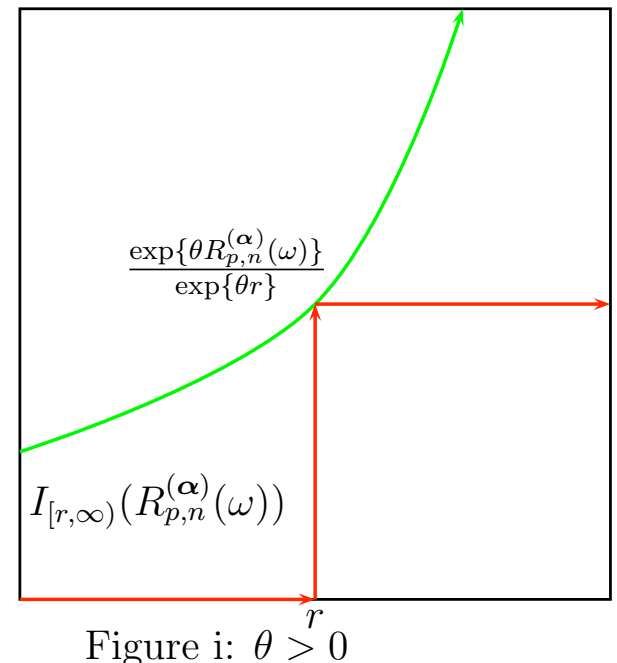

Figure i: $\theta>0$

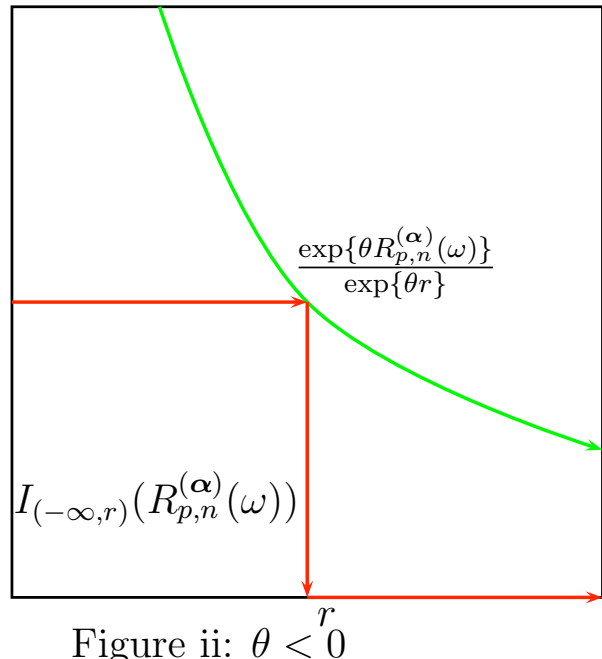

Figure ii: $\theta<0$

Furthermore, maximization of the RHS of (3) can be interpreted as the maximization of the expected value of the exponential utility function

$$
U\left(R_{p, n}^{(\boldsymbol{\alpha})}(\omega)\right)=-\left[\frac{\exp \left\{R_{p, n}^{(\boldsymbol{\alpha})}(\omega)\right\}}{\exp \{r\}}\right]^{\theta}
$$

which has the first and second derivatives

$$
\begin{aligned}
U^{\prime}\left(R_{p, n}^{(\boldsymbol{\alpha})}(\omega)\right) & =-\theta\left[\frac{\exp \left\{R_{p, n}^{(\boldsymbol{\alpha})}(\omega)\right\}}{\exp \{r\}}\right]^{\theta}, \\
U^{\prime \prime}\left(R_{p, n}^{(\boldsymbol{\alpha})}(\omega)\right) & =-\theta^{2}\left[\frac{\exp \left\{R_{p, n}^{(\boldsymbol{\alpha})}(\omega)\right\}}{\exp \{r\}}\right]^{\theta} .
\end{aligned}
$$


Hence, the Arrow-Pratt measure of absolute risk aversion is defined as

$$
\theta_{A P}=-\frac{U^{\prime \prime}\left(R_{p, n}^{(\boldsymbol{\alpha})}(\omega)\right)}{U^{\prime}\left(R_{p, n}^{(\boldsymbol{\alpha})}(\omega)\right)}=-\theta
$$

In particular, when $\tilde{r}_{i}$, with $i=1, . . n$ are IID, Theorem 1 states that the RHS of (II) or (3) can be made as small as possible so that it can adequately approximate the LHS as $(n)$ is sufficiently large.

Theorem 1. Let $r^{+}=\arg \lim _{n \longrightarrow \infty} \sup _{\mathbf{1}^{\prime} \boldsymbol{\alpha}=1} \Lambda_{n}(r, \boldsymbol{\alpha})=\infty$. Then, for $r \in\left(E\left[\tilde{r}_{1}\right], r^{+}\right)$and a given $\boldsymbol{\alpha}$ s.t. $\mathbf{1}^{\prime} \boldsymbol{\alpha}=1$ we have

$$
P\left\{R_{p, n}^{(\boldsymbol{\alpha})}(\omega)>r\right\} \simeq \frac{1}{\sqrt{2 \pi \sum_{1}^{n} \alpha_{i}^{2}} \theta(r) \sigma(r)} \exp \left\{-\Lambda_{n}(r, \boldsymbol{\alpha})\right\}
$$

where $R_{p, n}^{(\boldsymbol{\alpha})}(\omega)$ is defined in (1),$\Lambda_{n}(r, \boldsymbol{\alpha})=\sup _{\theta>0}\left\{r \theta-\log E\left[\exp \left\{\theta R_{p, n}^{(\boldsymbol{\alpha})}(\omega)\right\}\right]\right\}$ with $\theta(r)=$ $\arg \sup _{\theta>0}\left\{r \theta-\log E\left[\exp \left\{\theta R_{p, n}^{(\boldsymbol{\alpha})}(\omega)\right\}\right]\right\}$ and $\sigma^{2}(r)=\frac{1}{\theta^{\prime}(r)}$.

Similarly, let $r^{-}=\arg \lim _{n \longrightarrow \infty} \inf _{\mathbf{1}^{\prime} \boldsymbol{\alpha}=1} \Lambda_{n}(r, \boldsymbol{\alpha})=\infty$. Then, for $r \in\left(r^{-}, E\left[\tilde{r}_{1}\right]\right)$ we have

$$
P\left\{R_{p, n}^{(\boldsymbol{\alpha})}(\omega)<r\right\} \simeq \frac{1}{\sqrt{2 \pi \sum_{1}^{n} \alpha_{i}^{2}} \theta(r) \sigma(r)} \exp \left\{-\Lambda_{n}(r, \boldsymbol{\alpha})\right\}
$$

where $\Lambda_{n}(r, \boldsymbol{\alpha})=\sup _{\theta<0}\left\{r \theta-\log E\left[\exp \left\{\theta R_{p, n}^{(\boldsymbol{\alpha})}(\omega)\right\}\right]\right\}$ with $\theta(r)=\arg \sup _{\theta<0}\left\{r \theta-\log E\left[\exp \left\{\theta R_{p, n}^{(\boldsymbol{\alpha})}(\omega)\right\}\right]\right\}$ and $\sigma^{2}(r)=\frac{1}{\theta^{\prime}(r)}$.

Proof. See Appendix.

As shown in Remark 4.1 of Appendix A, in order to ensure that the deviations function for the lower tail probability/the upper tail probability has a unique maximal value, the benchmark $(r)$ in Theorem 1 must be set below/above the mean value $E\left[R_{p, n}^{(\boldsymbol{\alpha})}(\omega)\right]$.

However, when there are two risky assets, $\Lambda_{2}(r, \theta, \alpha)$ is concave in both $\alpha$ and $\theta$. This can be seen by applying an Edgeworth expansion (see e.g. Cramen, 1946) to the moment generating 
function (mgf), i.e.,

$$
\begin{aligned}
E\left[\exp \left\{\theta R_{p, n}^{(\boldsymbol{\alpha})}(\omega)\right\}\right] & =\exp \left\{\theta\left(\alpha E\left[\tilde{r}_{1}\right]+(1-\alpha) E\left[\tilde{r}_{2}\right]\right)+\frac{\theta^{2}}{2}\left(\alpha^{2} \operatorname{Var}\left[\tilde{r}_{1}\right]+(1-\alpha)^{2} \operatorname{Var}\left[\tilde{r}_{2}\right]\right.\right. \\
& \left.\left.+2 \alpha(1-\alpha) \operatorname{Cov}\left[\tilde{r}_{1}, \tilde{r}_{2}\right]\right)+ \text { remainder }\right\}
\end{aligned}
$$

In (4), the positions of $\theta$ and $\alpha$ can be exchanged without essentially changing the value of the deviations function as long as we have a finite truncation.

Therefore, maximization of the expected exponential utility (or the deviations function) is equivalent to minimization of the upper bound of the underperformance probability. That is

$$
\begin{aligned}
\min _{0<\alpha<1} P\left\{R_{p, n}^{(\boldsymbol{\alpha})}(\omega)<r\right\} & \lesssim-\max _{\substack{\theta<0 \\
0<\alpha<1}}-E\left[\frac{\exp \left\{R_{p, n}^{(\boldsymbol{\alpha})}(\omega)\right\}}{\exp \{r\}}\right]^{\theta} \\
& =\min _{\substack{\theta<0 \\
0<\alpha<1}} \exp \left\{-\Lambda_{2}(r, \theta, \alpha)\right\} \\
& =\exp \left\{-\max _{\substack{\theta<0 \\
0<\alpha<1}} \Lambda_{2}(r, \theta, \alpha)\right\} .
\end{aligned}
$$

Since the number of risky assets are small the RHS of (国), that is defined as the risk bound, may not be close enough to the LHS. Hence, the optimal portfolio ( $\hat{\alpha})$ found in (5) can be interpreted as the value that maximizes the expected exponential utility and minimizes the risk bound simultaneously.

Remark 2.1. By the large deviations principle in (Dembo and Zeitoun, 1999), the benchmark (r) in (5) must be set below $E\left[R_{p, n}^{(\boldsymbol{\alpha})}(\omega)\right]$ to ensure that a unique maximal value of the deviations function exist. Since $E\left[R_{p, n}^{(\boldsymbol{\alpha})}(\omega)\right]$ is the population mean that depends on a unknown quantity $(\alpha)$, the benchmark can empirically be set below the sample mean of a typical asset. Moreover, regarding the upper tail of the return distribution as in (21), (r) must be set above $E\left[R_{p, n}^{(\boldsymbol{\alpha})}(\omega)\right]$ for the existence of a unique maximal value.

Remark 2.2. As noted in (3), $\operatorname{Prob}\left\{R_{p, n}^{(\boldsymbol{\alpha})}(\omega)<r\right\}$ can be approximated by maximizing the deviations function w.r.t. $\alpha$ and $\theta$. Stutze (2004) advocates that the risk-aversion parameter $\theta_{A P}=-\theta$ can be determined by maximization of expected utility because the risk-aversion para- 
meter can be assumed to depend on a set of feasible investments. The mathematical logic behind this idea is owed to the Gartner-Ellis theorem (see e.g. Dembo and Zeitoun, 1999, page 45) that applies the Fenchel Legendre transformation to show that the RHS of (3) converges to the LHS when $(n)$ becomes large. However, by using asymmetry in the probability distribution of $R_{p, n}^{(\boldsymbol{\alpha})}(\omega)$, we can show a similar large deviations theorem without relying on the Fenchel Legendre transformation. Whereby, the conventional utility approach can be maintained; i.e., an expected utility function should be only partially maximized. In another word, the expected utility function can be maximized w.r.t. ( $\boldsymbol{\alpha})$ for any appropriately chosen risk-aversion parameter. Consequently, the risk bound in (国) is dependent on the risk aversion parameter $(\theta)$. This point is clarified in next sections.

\subsection{Implication of Asymmetry on Optimal Asset Allocation}

Recent studies in the empirical finance literature have reported many evidences of two types of asymmetry in equity returns. [See e.g. (Patton, 2004) and references therein.] The first is asymmetry in asset return distributions that leads to asymmetry in the portfolio return distribution, and the second is asymmetric dependence between two or more assets; i.e., asset returns appear to be more highly correlated during market downturns than during market upturns.

Now we provide a simple example just to illustrate the role played by asymmetric dependence and skewness in optimal investment problem. First, Let us define the first and second nonlinear correlations between two asset returns $\tilde{r}_{1}$ and $\tilde{r}_{2}$ as

$$
\begin{aligned}
\lambda_{12} & =\frac{E\left[\left[\tilde{r}_{1}-E\left(\tilde{r}_{1}\right)\right]\left[\tilde{r}_{2}-E\left(\tilde{r}_{2}\right)\right]^{2}\right]}{\sqrt{E\left[\tilde{r}_{1}-E\left(\tilde{r}_{1}\right)\right]^{2} E\left[\tilde{r}_{2}-E\left(\tilde{r}_{2}\right)\right]^{4}}}, \\
\lambda_{21} & =\frac{E\left[\left[\tilde{r}_{1}-E\left(\tilde{r}_{1}\right)\right]^{2}\left[\tilde{r}_{2}-E\left(\tilde{r}_{2}\right)\right]\right]}{\sqrt{E\left[\tilde{r}_{1}-E\left(\tilde{r}_{1}\right)\right]^{4} E\left[\tilde{r}_{2}-E\left(\tilde{r}_{2}\right)\right]^{2}}} .
\end{aligned}
$$

(See Appendix B for further details of $\lambda_{12}$ and $\lambda_{21}$.) Without losing generality, let us assume that $E\left[\tilde{r}_{1}\right]=E\left[\tilde{r}_{2}\right]=0$. In this case, an investor always prefers to hold a portfolio with positive 
skewness and probably lower variance. Moreover, let us define the skewness as

$$
\operatorname{skew}_{3}(\alpha)=\frac{\mu_{3}(\alpha)}{\mu_{2}^{3 / 2}(\alpha)},
$$

where

$$
\begin{aligned}
\mu_{3}(\alpha) & =E\left[\alpha \tilde{r}_{1}+(1-\alpha) \tilde{r}_{2}\right]^{3} \\
& =\alpha^{3} E\left[\tilde{r}_{1}^{3}\right]+(1-\alpha)^{3} E\left[\tilde{r}_{2}^{3}\right]+3 \alpha^{2}(1-\alpha) E\left[\tilde{r}_{1}^{2} \tilde{r}_{2}\right]+3 \alpha(1-\alpha)^{2} E\left[\tilde{r}_{1} \tilde{r}_{2}^{2}\right], \\
\mu_{2}(\alpha) & =E\left[\alpha \tilde{r}_{1}+(1-\alpha) \tilde{r}_{2}\right]^{2} \\
& =\alpha^{2} E\left[\tilde{r}_{1}^{2}\right]+(1-\alpha)^{2} E\left[\tilde{r}_{2}^{2}\right]+2 \alpha(1-\alpha) E\left[\tilde{r}_{1} \tilde{r}_{2}\right] .
\end{aligned}
$$

Suppose that $E\left[\tilde{r}_{1}^{3}\right]=E\left[\tilde{r}_{2}^{3}\right]=-1, E\left[\tilde{r}_{1}^{2}\right]=E\left[\tilde{r}_{2}^{2}\right]=1$ and $E\left[\tilde{r}_{1} \tilde{r}_{2}\right]=0$ we can compare investment strategies in two different cases as follows

1. Two assets are non-correlated, i.e. $E\left[\tilde{r}_{1}^{2} \tilde{r}_{2}\right]=E\left[\tilde{r}_{1} \tilde{r}_{2}^{2}\right]=0$, we shall examine two strategies

(a) $\alpha=0.5: \operatorname{skew}_{3}(0.5)=-0.714, \mu_{2}(0.5)=0.5$

(b) $\alpha=0.4: \operatorname{skew}_{3}(0.4)=-0.756, \mu_{2}(0.4)=0.52$.

Since Strategy (a) has a lower variance and a higher skewness, thus Strategy (a) is obviously preferred to Strategy (b).

2. Two assets are non-linearly correlated, i.e. $E\left[\tilde{r}_{1}^{2} \tilde{r}_{2}\right]=-0.3$ and $E\left[\tilde{r}_{1} \tilde{r}_{2}^{2}\right]=0.9$, we shall examine two strategies

(a) $\alpha=0.5: \operatorname{skew}_{3}(0.5)=-0.071, \mu_{2}(0.5)=0.5$

(b) $\alpha=0.4: \operatorname{skew}_{3}(0.4)=0.06, \mu_{2}(0.4)=0.52$.

Strategy (b) might be preferred to Strategy (a). Since a typical investor prefers positive skewness and dislikes high variance, he/she will select Strategy (b) if he/she is more concerned about negative skewness. 
Hence, it is interesting to examine the implication of asymmetry in a portfolio return distribution and asymmetric dependence on optimal asset allocation. First, a parametric model is proposed to capture the asymmetric characteristics of portfolio return distribution. Next, an Edgeworth expansion can be applied to pick out the nonlinear correlations of asset returns $\left(\lambda_{12}, \lambda_{21}\right)$, whereby we can examine their effects on an optimal portfolio choice and its risk bound.

\subsection{Parametric Implementation}

\subsubsection{Large portfolio optimization technique}

The deviations function in (5) is the Fenchel-Legendre transformation of the logarithm of the mgf. If individual returns are uncorrelated, the mgf of the portfolio return is simply the product of the individual mgfs, thus no assumption on the portfolio return distribution is necessary. A standard optimization technique can then be applied to maximize the deviations function. However, when individual returns are not uncorrelated, it is necessary to impose an explicit assumption on the portfolio return distribution. This portfolio optimization technique is based on 1) maximizing the expected log likelihood function of the portfolio return 2) maximizing the deviations function in (国) w.r.t. the investment proportion $(\boldsymbol{\alpha})$ for a given degree of absolute risk aversion. Note that the degree of absolute risk aversion should be chosen in the vicinity of the maximal values $\left(\theta^{*}, \boldsymbol{\alpha}^{*}\right)$ of the deviations function so that the RHS of (国) can be tightened to the LHS by an asymptotic argument.

In another word, maximization of an expected log likelihood function, that is interpreted as the minus of the empirical entropy of the portfolio return, is equivalent to minimization of the uncertainty level of the portfolio return. Moreover, as shown in (3), maximization of a deviations function is equivalent to maximization of an expected utility function. Both can be associated with minimization of the risk that the portfolio return may fall below an investable benchmark.

Suppose that there are $(n)$ risky assets, the portfolio return is given by

$$
\begin{aligned}
R_{p, n}^{(\boldsymbol{\alpha})}(\omega) & =\sum_{i=1}^{n-1} \alpha_{i} \tilde{r}_{i}+\left(1-\sum_{i=1}^{n-1} \alpha_{i}\right) \tilde{r}_{n} \\
& =\boldsymbol{\alpha}^{\prime} \tilde{\mathbf{r}}+\left(1-\mathbf{1}^{\prime} \boldsymbol{\alpha}\right) \tilde{r}_{n}
\end{aligned}
$$


where $\boldsymbol{\alpha}^{\prime}=\left\{\alpha_{1}, . ., \alpha_{n-1}\right\}$ and $\tilde{\mathbf{r}}^{\prime}=\left\{\tilde{r}_{1}, . ., \tilde{r}_{n-1}\right\}$.

Let us assume that $R_{p, n}^{(\boldsymbol{\alpha})}(\omega) \sim p d f\left(R_{p, n}^{(\boldsymbol{\alpha})}(\omega) \mid \Theta\right)$, where $R_{p, n}^{(\boldsymbol{\alpha})}(\omega)$ stresses that $R_{p}$ depends on $(\boldsymbol{\alpha})$ and $(n)$. Given a sample of size $T\left\{\tilde{\mathbf{r}}_{t}\right\}_{t=1}^{T}$, the log-likelihood function can be written as

$$
\ell(\Theta, \boldsymbol{\alpha})=\frac{1}{T} \sum_{t=1}^{T} \log p d f\left(R_{p, n, t}^{(\boldsymbol{\alpha})}(\omega) \mid \Theta\right) .
$$

Therefore, the ML estimate of $\Theta$ is

$$
\widehat{\Theta}(\boldsymbol{\alpha})=\arg \max _{\Theta \in A} \ell(\Theta, \boldsymbol{\alpha})
$$

for a given $\boldsymbol{\alpha}$, where $A$ is a compact set containing the true parameters, and $\widehat{\Theta}(\boldsymbol{\alpha})$ stresses that the ML estimate depends on a specific $\boldsymbol{\alpha}$. The ideal case is when $\widehat{\Theta}(\boldsymbol{\alpha})$ is a function of $\boldsymbol{\alpha}$. Otherwise, we can rely on an interpolation technique to find an approximation of $\widehat{\Theta}(\boldsymbol{\alpha})$ that is a polynomial of $(\boldsymbol{\alpha})$.

Furthermore, in view of (5) it can be shown that as the number of risky assets $(n)$ is large, we have

$$
\begin{aligned}
\log P\left\{R_{p, n}^{(\boldsymbol{\alpha})}(\omega)<r\right\} & \approx-\max _{\substack{\theta<0 \\
\boldsymbol{\alpha} \in S}}\left[r \theta-\log \exp \left\{\theta R_{p, n}^{(\boldsymbol{\alpha})}(\omega)\right\}\right] \\
& =-\max _{\substack{\theta<0 \\
\boldsymbol{\alpha} \in S}} \Lambda_{n}(r, \theta, \widehat{\Theta}(\boldsymbol{\alpha}))
\end{aligned}
$$

where $S$ is the set of feasible investments. (The formal result for asymmetric return distributions will be given in the next section.)

For a given risk-aversion parameter $(\hat{\theta}<0)$ in the vicinity of $\left(\theta^{*}\right)$ that is obtained by total maximization of $\Lambda_{n}(r, \theta, \widehat{\Theta}(\boldsymbol{\alpha}))$, the optimal portfolio is given as

$$
\hat{\boldsymbol{\alpha}}(\hat{\theta})=\arg \max _{\boldsymbol{\alpha} \in S} \Lambda_{n}(r, \hat{\theta}, \widehat{\Theta}(\boldsymbol{\alpha}))
$$


$\Leftrightarrow$

$$
\hat{\boldsymbol{\alpha}}(\hat{\theta})=\arg \left\{\boldsymbol{\alpha}:\left.\frac{\partial \Lambda(r, \hat{\theta}, \Theta)}{\partial \Theta}\right|_{\Theta=\widehat{\Theta}(\boldsymbol{\alpha})} \frac{\partial \widehat{\Theta}(\boldsymbol{\alpha})}{\partial \boldsymbol{\alpha}}=0\right\} .
$$

Suppose that the distributions of $\tilde{\mathbf{r}}$ are $p d f\left(\tilde{r}_{i}\right)$ with the central moments $\mu_{i, m}=E\left[\tilde{r}_{i}-E\left[\tilde{r}_{i}\right]\right]^{m}$ for $i=1, . ., n$, then we have the following relationship

$$
\begin{aligned}
& E\left[R_{p, n}^{(\hat{\boldsymbol{\alpha}})}(\omega)-E\left[R_{p, n}^{(\hat{\boldsymbol{\alpha}})}(\omega)\right]\right]^{m}=E\left[\sum_{i=1}^{n}\left(\tilde{r}_{i}-\mu_{i, 1}\right)\right]^{m} \\
& =E\left[\sum_{\substack{i=1 \\
\sum_{k=1}^{i} m_{k}=m}}^{n}\left(\begin{array}{c}
m \\
m_{1}, \ldots, m_{i}
\end{array}\right) \prod_{j=1}^{j=i}\left[\hat{\alpha}_{j}\left(\tilde{r}_{j}-\mu_{j, 1}\right)\right]^{m_{j}}\right] \\
& =\sum_{i=1}^{n} \hat{\alpha}_{i}^{m} E\left[\tilde{r}_{i}-E\left[\tilde{r}_{i}\right]\right]^{m}
\end{aligned}
$$

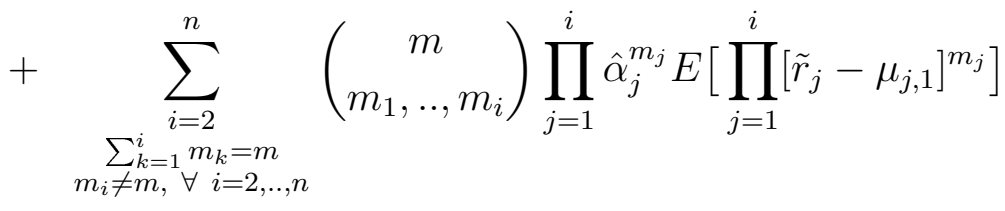

$$
\begin{aligned}
& =\sum_{i=1}^{n} \hat{\alpha}_{i}^{m} \mu_{i, m}+\sum_{\substack{i=2 \\
\sum_{k=1}^{i} m_{k}=m \\
m_{i} \neq m, \forall i=2, . ., n}}^{n}\left(\begin{array}{c}
m \\
m_{1}, . ., m_{i}
\end{array}\right) \prod_{j=1}^{i} \hat{\alpha}_{j}^{m_{j}} \rho_{m_{1}, . . m_{i}},
\end{aligned}
$$

where $\rho_{m_{1}, . . m_{i}} \forall i=2, . ., n$ are the measures of covariance.

Hence, for an optimal portfolio as given in (7), the individual central moments, and the central moments of the pdf of $R_{p, n}^{\hat{\alpha}}(\omega)$ that is estimated by (6), the relationship among the measures of correlation that is denoted by the second term in the RHS of (8) can be recovered by using (8). Although this technique does not enable us to effectively recover the individual values of the high order measures of correlation, an interesting implication of (8) is that although the individual nonlinear correlations may not effect the optimal portfolio and the risk, an appropriate linear combination of the individual linear correlation or the nonlinear ones, that contributes to a decrease in the portfolio variance or an increase in the portfolio skewness, can have a strong impact on the optimal portfolio and the risk. We will address this further in the empirical analysis.

Note that when individual asset returns are independent, the probability distribution of the 
portfolio return $R_{p, n}^{(\boldsymbol{\alpha})}(\omega)$ can be straightforwardly derived from the distributions of the individual returns. For instance, the weighted sums of Normal, Gamma, or Chi squared random variables are Normal, Gamma, or Chi squared distributed respectively. (See Rad, 1973 for the proofs.) However, when asset returns are dependent, it is rather complicated to derive the distribution of the portfolio return that is usually dependent on a specific assumption about dependence. Thus, in this section we assume a certain parametric form for the portfolio return distribution that depends on an investable portfolio $(\boldsymbol{\alpha})$. As a consequence, the measures of covariance can be recovered from the relationship (8), thus explicitly depend on the optimal portfolio ( $\hat{\boldsymbol{\alpha}})$. An explanation is that the probabilities of the extreme values of $R_{p, n}^{(\boldsymbol{\alpha})}(\omega)$ are so small that they contribute negligibly to the value of the average log-likelihood function (6). Thus, it is sensible to choose a proportion $(\hat{\boldsymbol{\alpha}})$ such that the frequency of realizations of $R_{p, n}^{(\boldsymbol{\alpha})}(\omega)$ in the lower tail of the portfolio return distribution is as small as possible. This is consequently equivalent to selecting a portfolio $(\hat{\boldsymbol{\alpha}})$ such that the underperformance probability is minimized whilst the expected utility function is maximized. This idea can be summarized in the following remark.

Remark 2.3. The optimal portfolio $(\hat{\boldsymbol{\alpha}})$ is the maximal value of the deviations function and the minimal value of the frequency of the lower tail extreme values of $R_{p, n}^{(\boldsymbol{\alpha})}(\omega)$. Thus, the recovered measures of covariance are dependent on $\hat{\boldsymbol{\alpha}}$.

In order to illustrate how this technique works, let us assume that there are two risky assets; and that the portfolio return is normal distributed, i.e.,

$$
\begin{aligned}
p d f\left(R_{p, 2}^{(\alpha)}(\omega)\right) & =\frac{1}{\sqrt{2 \pi} \sigma_{p}} \exp \left\{-\frac{1}{2}\left[\frac{R_{p, 2}^{(\alpha)}(\omega)-\mu_{p}}{\sigma_{p}}\right]^{2}\right\} \\
m g f(\theta) & =\exp \left\{\theta \mu_{p}+\frac{\theta^{2}}{2} \sigma_{p}^{2}\right\} .
\end{aligned}
$$


Then, the ML estimates of $\mu_{p}$ and $\sigma_{p}$ are

$$
\begin{aligned}
\hat{\mu}_{p}(\alpha) & =\frac{1}{T} \sum_{t=1}^{T} R_{p, 2}^{(\alpha)}(\omega), \\
\hat{\sigma}_{p}^{2}(\alpha) & =\frac{1}{T} \sum_{t=1}^{T}\left(R_{p, 2}^{(\alpha)}(\omega)-\hat{\mu}_{p}(\alpha)\right)^{2}, \\
\frac{\partial \hat{\mu}_{p}(\alpha)}{\partial \alpha} & =\frac{1}{T} \sum_{t=1}^{T}\left(\tilde{r}_{1 t}-\tilde{r}_{2 t}\right), \\
\frac{\partial \hat{\sigma}_{p}^{2}(\alpha)}{\partial \alpha} & =\frac{2}{T} \sum_{t=1}^{T}\left(R_{p, 2}^{(\alpha)}(\omega)-\hat{\mu}_{p}(\alpha)\right)\left(\tilde{r}_{1, t}-\tilde{r}_{2, t}\right) .
\end{aligned}
$$

Let $\theta^{*}$ denotes the maximal value obtained by maximizing $\Lambda_{n}(r, \theta, \alpha)$ w.r.t. $\alpha$ and $\theta$. Then, for a given $\hat{\theta}$ in the neighbourhood of $\theta^{*} \hat{\alpha}(\hat{\theta})$ is a solution to

$$
\hat{\theta} \frac{\partial \hat{\mu}(\alpha)}{\partial \alpha}+\frac{\hat{\theta}^{2}}{2} \frac{\partial \hat{\sigma}^{2}(\alpha)}{\partial \alpha}=0
$$

Hence, we have

$$
\hat{\alpha}(\hat{\theta})=-\frac{A}{B}
$$

where $A$ and $B$ are defined as

$$
\begin{aligned}
& A=\hat{\theta}\left\{\frac{1}{T}\left(2-\frac{1}{T}\right)\left[\sum_{t=1}^{T}\left(\tilde{r}_{1 t}-\tilde{r}_{2 t}\right)\right]^{2}-\sum_{t=1}^{T}\left(\tilde{r}_{1 t}-\tilde{r}_{2 t}\right)^{2}\right\}-\sum_{t=1}^{T}\left(\tilde{r}_{1 t}-\tilde{r}_{2 t}\right), \\
& B=\hat{\theta}\left\{\sum_{t=1}^{T} \tilde{r}_{2 t}\left(\tilde{r}_{1 t}-\tilde{r}_{2 t}\right)-\frac{T^{2}-T-1}{T} \sum_{t=1}^{T} \tilde{r}_{2 t} \sum_{t=1}^{T}\left(\tilde{r}_{1 t}-\tilde{r}_{2 t}\right)\right\} .
\end{aligned}
$$

\subsubsection{Asymmetric Gamma distribution}

To capture asymmetry in a return distribution, we can use a mixture of two Gamma distributions. This distribution basically represents that an asset return can be positive or negative with unequal likelihoods. Furthermore, asset returns are more correlated during a trough than a boom. As a result, a portfolio return distribution must have some sorts of asymmetry. The asymmetric Gamma distribution is proposed by Knight et a1. (1995) as a method to capture asymmetry in 
asset returns. In view of Knight et al. (1995), our analysis is based on two cases; 1) asset returns are uncorrelated, and 2) asset returns are correlated. First, we state the statistical properties of the asymmetric Gamma distribution and the (partially) symmetric Gamma distribution.

\section{The Asymmetric Gamma Distribution}

A return distribution is a mixture of two Gamma distributions, i.e.,

$$
\tilde{r} \sim \operatorname{mixture}\left(p, \operatorname{Gamma}\left(\gamma_{1}, \kappa_{1}\right), \operatorname{Gamma}\left(\gamma_{2}, \kappa_{2}\right)\right)
$$

if its values depend on whether return is positive or negative. The probability density is given by

$p d f(\tilde{r})=p I_{[0, \infty)}(\tilde{r}) \frac{1}{\gamma_{1}^{\kappa_{1}} \Gamma\left(\kappa_{1}\right)} \tilde{r}^{\kappa_{1}-1} \exp \left\{-\frac{\tilde{r}}{\gamma_{1}}\right\}+(1-p) I_{(-\infty, 0)}(\tilde{r}) \frac{1}{\gamma_{2}^{\kappa_{2}} \Gamma\left(\kappa_{2}\right)}(-\tilde{r})^{\kappa_{2}-1} \exp \left\{\frac{\tilde{r}}{\gamma_{2}}\right\}$

where $\gamma_{1}$ and $\gamma_{2}$ are positive. $\Gamma(\bullet)$ is the standard Gamma function.

Given a sample of size $T\left\{\tilde{r}_{t}\right\}_{t=1}^{T}$, the likelihood function can be written as

$$
\begin{aligned}
\prod_{t=1}^{T} p d f\left(\tilde{r}_{t}\right)=\prod_{t=1}^{T}\left\{p I_{[0, \infty)}\left(\tilde{r}_{t}\right) \frac{1}{\gamma_{1}^{\kappa_{1}} \Gamma\left(\kappa_{1}\right)} \tilde{r}_{t}^{\kappa_{1}-1} \exp \left\{-\frac{\tilde{r}_{t}}{\gamma_{1}}\right\}\right. & +(1-p) I_{(-\infty, 0)}\left(\tilde{r}_{t}\right) \\
& \left.\frac{1}{\gamma_{2}^{\kappa_{2}} \Gamma\left(\kappa_{2}\right)}\left(-\tilde{r}_{t}\right)^{\kappa_{2}-1} \exp \left\{\frac{\tilde{r}_{t}}{\gamma_{2}}\right\}\right\} .
\end{aligned}
$$

Since the RHS of (9) is the product of sums of the orthogonal terms, we have

$$
\begin{array}{r}
\prod_{t=1}^{T} p d f\left(\tilde{r}_{t}\right)=p^{\#\left\{t \in[1, T]: \tilde{r}_{t} \geq 0\right\}}(1-p)^{T-\#\left\{t \in[1, T]: \tilde{r}_{t} \geq 0\right\}} \prod_{t \in\left\{t \in[1, T]: \tilde{r}_{t} \geq 0\right\}}\left\{I_{[0, \infty)}\left(\tilde{r}_{t}\right) \frac{1}{\gamma_{1}^{\kappa_{1}} \Gamma\left(\kappa_{1}\right)}\right. \\
\left.\tilde{r}_{t}^{\kappa_{1}-1} \exp \left\{-\frac{\tilde{r}_{t}}{\gamma_{1}}\right\}\right\} \prod_{t \in\left\{t \in[1, T]: \tilde{r}_{t}<0\right\}}\left\{I_{(-\infty, 0)}\left(\tilde{r}_{t}\right) \frac{1}{\gamma_{2}^{\kappa_{2}} \Gamma\left(\kappa_{2}\right)}\left(-\tilde{r}_{t}\right)^{\kappa_{2}-1} \exp \left\{\frac{\tilde{r}_{t}}{\gamma_{2}}\right\}\right\}
\end{array}
$$

where $\#\left\{t \in[1, T]: \tilde{r}_{t} \geq 0\right\}$ is the number of the nonnegative return realizations in the sample.

Since the shifting probability $(p)$ has the sample estimate $\hat{p}=\frac{\#\left\{t \in[1, T]: \tilde{r}_{t} \geq 0\right\}}{T}$, the log likeli- 
hood function can be written as

$$
\begin{aligned}
\ell\left(\gamma_{1}, \gamma_{2}, \kappa_{1}, \kappa_{2}\right) & =\#\left\{t \in[1, T]: \tilde{r}_{t} \geq 0\right\} \log (\hat{p})+\left(T-\#\left\{t \in[1, T]: \tilde{r}_{t}>0\right\}\right) \log (1-\hat{p}) \\
& +\left(\kappa_{1}-1\right) \sum_{t \in\left\{t \in[1, T]: \tilde{r}_{t} \geq 0\right\}} \log \left(\tilde{r}_{t} I_{[0, \infty)}\left(\tilde{r}_{t}\right)\right)-\frac{1}{\gamma_{1}} \sum_{t \in\left\{t \in[1, T]: \tilde{r}_{t} \geq 0\right\}} \tilde{r}_{t} I_{[0, \infty)}\left(\tilde{r}_{t}\right) \\
& -\#\left\{t \in[1, T]: \tilde{r}_{t} \geq 0\right\}\left(\kappa_{1} \log \left(\gamma_{1}\right)+\log \left(\Gamma\left(\kappa_{1}\right)\right)\right) \\
& +\left(\kappa_{2}-1\right) \sum_{t \in\left\{t \in[1, T]: \tilde{r}_{t}<0\right\}} \log \left(-\tilde{r}_{t} I_{(-\infty, 0)}\left(\tilde{r}_{t}\right)\right)+\frac{1}{\gamma_{2}} \sum_{t \in\left\{t \in[1, T]: \tilde{r}_{t}<0\right\}} \tilde{r}_{t} I_{(-\infty, 0)}\left(\tilde{r}_{t}\right) \\
& -\#\left\{t \in[1, T]: \tilde{r}_{t}<0\right\}\left(\kappa_{2} \log \left(\gamma_{2}\right)+\log \left(\Gamma\left(\kappa_{2}\right)\right)\right) .
\end{aligned}
$$

By taking derivatives of $\ell\left(\gamma_{1}, \gamma_{2}, \kappa_{1}, \kappa_{2}\right)$ w.r.t. $\left(\gamma_{1}, \gamma_{2}, \kappa_{1}, \kappa_{2}\right)$, the ML estimates $\left(\hat{\gamma_{1}}, \hat{\gamma_{2}}, \hat{\kappa_{1}}, \hat{\kappa_{2}}\right)$ are the solutions to

$$
\left\{\begin{aligned}
\frac{\Gamma^{\prime}\left(\kappa_{1}\right)}{\Gamma\left(\kappa_{1}\right)}-\log \left(\kappa_{1}\right) & =\frac{\sum_{t=1}^{T} \log \left(\tilde{r}_{t} I_{[0, \infty)}\left(\tilde{r}_{t}\right)\right)}{\#\left\{t \in[1, T]: \tilde{r}_{t} \geq 0\right\}}-\log \left(\sum_{t=1}^{T} \frac{\tilde{r}_{t} I_{[0, \infty)}\left(\tilde{r}_{t}\right)}{\#\left\{t \in[1, T]: \tilde{r}_{t} \geq 0\right\}}\right) \\
\frac{\Gamma^{\prime}\left(\kappa_{2}\right)}{\Gamma\left(\kappa_{2}\right)}-\log \left(\kappa_{2}\right) & =\frac{\sum_{t=1}^{T} \log \left(-\tilde{r}_{t} I_{(-\infty, 0)}\left(\tilde{r}_{t}\right)\right)}{\#\left\{t \in[1, T]: \tilde{r}_{t}<0\right\}}-\log \left(-\frac{\sum_{t=1}^{T} \tilde{r}_{t} I_{(-\infty, 0)}\left(\tilde{r}_{t}\right)}{\#\left\{t \in[1, T]: \tilde{r}_{t}<0\right\}}\right) \\
\gamma_{1} & =\frac{\sum_{t=1}^{T} \tilde{r}_{t} I_{[0, \infty)}\left(\tilde{r}_{t}\right)}{\#\left\{t \in[1, T]: \tilde{r}_{t} \geq 0\right\} \kappa_{1}} \\
\gamma_{2} & =-\frac{\sum_{t=1}^{T} \tilde{r}_{t} I_{(-\infty, 0)}\left(\tilde{r}_{t}\right)}{\#\left\{t \in[1, T]: \tilde{r}_{t}<0\right\} \kappa_{2}}
\end{aligned}\right.
$$

Figure 2 shows that the functions $f(\kappa)=$ the LHS - the RHS of the first two equations in (10) are increasing, thus the ML estimates are the unique solutions to (10).

The asymptotic variances of $\left(\hat{\gamma_{1}}, \hat{\gamma_{2}}, \hat{\kappa_{1}}, \hat{\kappa_{2}}\right)$ are the diagonal elements of the inverse of the sample information matrix as given by

$$
\mathbf{I}=T \operatorname{inv}\left(\begin{array}{cccc}
P_{\kappa_{1}^{2}}^{*} & P_{\kappa_{1} \gamma_{1}}^{*} & P_{\kappa_{1} \gamma_{2}}^{*} & P_{\kappa_{1}, \kappa_{2}}^{*} \\
P_{\gamma_{1} \kappa_{1}}^{*} & P_{\gamma_{1}^{2}}^{*} & P_{\gamma_{1} \gamma_{2}}^{*} & P_{\gamma_{1} \kappa_{2}}^{*} \\
P_{\gamma_{2} \kappa_{1}}^{*} & P_{\gamma_{2} \gamma_{1}}^{*} & P_{\gamma_{2}^{2}}^{*} & P_{\gamma_{2} \kappa_{2}}^{*} \\
P_{\kappa_{2} \kappa_{1}}^{*} & P_{\kappa_{2} \gamma_{1}}^{*} & P_{\kappa_{2} \gamma_{2}}^{*} & P_{\kappa_{2}^{2}}^{*}
\end{array}\right)_{\left(\gamma_{1}, \gamma_{2}, \kappa_{1}, \kappa_{2}\right)=\left(\hat{\gamma_{1}}, \hat{\gamma_{2}}, \hat{\kappa_{1}}, \hat{\hat{\kappa}_{2}}\right)},
$$


where

$$
\begin{aligned}
P_{\gamma_{1}^{2}}^{*} & =\#\left\{t \in[1, T]: \tilde{r}_{t} \geq 0\right\} \frac{\kappa_{1}}{\gamma_{1}^{2}}-\frac{2}{\gamma_{1}^{3}} \sum_{t=1}^{T} \tilde{r}_{t} I_{[0, \infty)}\left(\tilde{r}_{t}\right), \\
P_{\gamma_{2}^{2}}^{*} & =\#\left\{t \in[1, T]: \tilde{r}_{t}<0\right\} \frac{\kappa_{2}}{\gamma_{2}^{2}}+\frac{2}{\gamma_{2}^{3}} \sum_{t=1}^{T} \tilde{r}_{t} I_{(-\infty, 0)}\left(\tilde{r}_{t}\right), \\
P_{\kappa_{1}^{2}}^{*} & =-\#\left\{t \in[1, T]: \tilde{r}_{t} \geq 0\right\} \frac{\Gamma^{\prime \prime}\left(\kappa_{1}\right) \Gamma\left(\kappa_{1}\right)-\left(\Gamma^{\prime}\left(\kappa_{1}\right)^{2}\right)}{\Gamma^{2}\left(\kappa_{1}\right)}, \\
P_{\kappa_{2}^{2}}^{*} & =-\#\left\{t \in[1, T]: \tilde{r}_{t}<0\right\} \frac{\Gamma^{\prime \prime}\left(\kappa_{2}\right) \Gamma\left(\kappa_{2}\right)-\left(\Gamma^{\prime}\left(\kappa_{2}\right)^{2}\right)}{\Gamma^{2}\left(\kappa_{2}\right)} \\
P_{\gamma_{1} \kappa_{1}}^{*} & =-\#\left\{t \in[1, T]: \quad \tilde{r}_{t} \geq 0\right\} \frac{1}{\gamma_{1}}, \\
P_{\gamma_{2} \kappa_{1}}^{*} & =-\#\left\{t \in[1, T]: \tilde{r}_{t} \geq 0\right\} \frac{1}{\gamma_{2}}, \\
P_{\gamma_{1} \kappa_{2}}^{*} & =P_{\gamma_{1} \gamma_{2}}^{*}\left(=P_{\kappa_{1} \gamma_{2}}^{*}=0\right)
\end{aligned}
$$

where $\Gamma^{\prime}$ and $\Gamma^{\prime \prime}$ are the first and the second derivatives of the standard Gamma function.

2. The (Partially) Symmetric Gamma Distribution (i.e. $\gamma_{1}=\gamma_{2}$ )

Let us assume that the scale parameters $\left(\gamma_{1}, \gamma_{2}\right)$ are equal. Thus, in this case the moments become more symmetric than the other case. The case of complete asymmetry (i.e., both the scale parameters and the magnitude ones are the same) can be derived straightforwardly. Therefore, by applying the conventional MLE method, the ML estimates $\left(\hat{\gamma}, \hat{\hat{\kappa}}_{1}, \hat{\hat{\kappa}}_{2}\right)$ for the partially symmetric Gamma distribution are the solutions to

$$
\left\{\begin{aligned}
& \frac{\Gamma^{\prime}\left(\kappa_{1}\right)}{\Gamma\left(\kappa_{1}\right)}-\log \left(\#\left\{t \in[1, T]: \tilde{r}_{t} \geq 0\right\} \kappa_{1}+\#\left\{t \in[1, T]: \tilde{r}_{t}<0\right\} \kappa_{2}\right) \\
= & \frac{1}{\#\left\{t \in[1, T]: \tilde{r}_{t} \geq 0\right\}} \sum_{t=1}^{T} \log \left(\tilde{r}_{t} I_{[0, \infty)}\left(\tilde{r}_{t}\right)\right)-\log \sum_{t=1}^{T} \tilde{r}_{t}\left(I_{(0, \infty)}\left(\tilde{r}_{t}\right)-I_{(-\infty, 0)}\left(\tilde{r}_{t}\right)\right), \\
& \frac{\Gamma^{\prime}\left(\kappa_{2}\right)}{\Gamma\left(\kappa_{2}\right)}-\log \left(\#\left\{t \in[1, T]: \tilde{r}_{t} \geq 0\right\} \kappa_{1}+\#\left\{t \in[1, T]: \tilde{r}_{t}<0\right\} \kappa_{2}\right) \\
= & \frac{1}{\#\left\{t \in[1, T]: \tilde{r}_{t}<0\right\}} \sum_{t=1}^{T} \log \left(-\tilde{r}_{t} I_{(-\infty, 0)}\left(\tilde{r}_{t}\right)\right)-\log \sum_{t=1}^{T} \tilde{r}_{t}\left(I_{(0, \infty)}\left(\tilde{r}_{t}\right)-I_{(-\infty, 0)}\left(\tilde{r}_{t}\right)\right), \\
\gamma= & \frac{\sum_{t=1}^{T} \tilde{r}_{t}\left(I_{(0, \infty)}\left(\tilde{r}_{t}\right)-I_{(-\infty, 0)}\left(\tilde{r}_{t}\right)\right)}{\#\left\{t \in[1, T]: \tilde{r}_{t} \geq 0\right\} \kappa_{1}+\#\left\{t \in[1, T]: \tilde{r}_{t}<0\right\} \kappa_{2}} .
\end{aligned}\right.
$$

The asymptotic variances of $\left(\hat{\gamma}, \hat{\hat{\kappa}}_{1}, \hat{\hat{\kappa}}_{2}\right)$ are the diagonal elements of the inverse of the sample 
information matrix as given by

$$
\mathbf{I}=T \operatorname{inv}\left(\begin{array}{ccc}
P_{\kappa_{1}^{2}}^{*} & P_{\kappa_{1} \gamma}^{*} & P_{\kappa_{1}, \kappa_{2}}^{*} \\
P_{\gamma \kappa_{1}}^{*} & P_{\gamma^{2}}^{*} & P_{\gamma \kappa_{2}}^{*} \\
P_{\kappa_{2} \kappa_{1}}^{*} & P_{\kappa_{2} \gamma}^{*} & P_{\kappa_{2}^{2}}^{*}
\end{array}\right)_{\left(\gamma, \kappa_{1}, \kappa_{2}\right)=\left(\hat{\gamma}, \hat{\kappa}_{1}, \hat{\kappa}_{2}\right)}
$$

where

$$
\begin{aligned}
P_{\gamma^{2}}^{*} & =-\frac{2}{\gamma^{3}} \sum_{t=1}^{T} \tilde{r}_{t}\left(I_{(0, \infty)}\left(\tilde{r}_{t}\right)-I_{(-\infty, 0)}\left(\tilde{r}_{t}\right)\right)+\#\left\{t \in[1, T]: \tilde{r}_{t} \geq 0\right\} \frac{\kappa_{1}}{\gamma^{2}} \\
P_{\gamma \kappa_{1}}^{*} & =-\#\left\{t \in[1, T]: \tilde{r}_{t} \geq 0\right\} \frac{1}{\gamma} \\
P_{\gamma \kappa_{2}}^{*} & =-\#\left\{t \in[1, T]: \quad \tilde{r}_{t}<0\right\} \frac{1}{\gamma}, \\
P_{\kappa_{1}^{2}}^{*} & =-\#\left\{t \in[1, T]: \quad \tilde{r}_{t} \geq 0\right\} \frac{\Gamma^{\prime \prime}\left(\kappa_{1}\right) \Gamma\left(\kappa_{1}\right)-\left(\Gamma^{\prime}\left(\kappa_{1}\right)\right)^{2}}{\Gamma^{2}\left(\kappa_{1}\right)}, \\
P_{\kappa_{2}^{2}}^{*} & =-\#\left\{t \in[1, T]: \tilde{r}_{t}<0\right\} \frac{\Gamma^{\prime \prime}\left(\kappa_{2}\right) \Gamma\left(\kappa_{2}\right)-\left(\Gamma^{\prime}\left(\kappa_{2}\right)\right)^{2}}{\Gamma^{2}\left(\kappa_{2}\right)} \\
P_{\kappa_{1} \kappa_{2}}^{*} & =0 .
\end{aligned}
$$

The likelihood ratio (LR) test for the null hypothesis of partial asymmetry against the alternative one of symmetry (i.e., $\gamma_{1}=\gamma_{2}$ ) can be formulated as

$$
L R=2\left(\ell\left(\hat{\gamma}_{1}, \hat{\gamma}_{2}, \hat{\kappa}_{1}, \hat{\kappa}_{2}\right)-\ell\left(\hat{\gamma}, \hat{\hat{\kappa}}_{1}, \hat{\hat{\kappa}}_{2}\right)\right) \sim \chi^{2}(1)
$$

where $\chi^{2}(1)$ is the Chi squared distribution with 1 degree of freedom.

Next, let us assume that the portfolio return $\left(R_{p, n}^{(\boldsymbol{\alpha})}(\omega)\right)$ has the asymmetric Gamma distribution. We state Theorem which shows that when the portfolio return distribution is asymmetric such that the probability that the portfolio return is in the vicinity of zero is arbitrary small, the RHS of (国) or (3) can be tightened as close as possible to the LHS. The theorem does not require the deviations function totally maximized w.r.t. the investable proportions $(\boldsymbol{\alpha})$ and the risk aversion parameter $(\theta)$. [See Stutzer (2003) for further details.] However, the risk aver- 
sion parameter is required to remain in the neighbourhood of the totally maximal value of the deviations function.

Theorem 2. Suppose that the return of a portfolio of $(n)$ risky assets

$$
R_{p, n}^{(\boldsymbol{\alpha})}(\omega)=\boldsymbol{\alpha}^{\prime} \tilde{\mathbf{r}}+\left(1-\mathbf{1}^{\prime} \boldsymbol{\alpha}\right) \tilde{r}_{n}
$$

where $\boldsymbol{\alpha}^{\prime}=\left(\alpha_{1}, . ., \alpha_{n-1}\right)$ and $\tilde{\mathbf{r}}^{\prime}=\left(\tilde{r}_{1}, . ., \tilde{r}_{n-1}\right)$, is asymmetrically distributed, we have

$$
\lim _{n \longrightarrow \infty} \frac{1}{n} \sup _{\boldsymbol{\alpha} \in S} \log \left(P\left(R_{p, n}^{(\boldsymbol{\alpha})}(\omega)>r\right)\right)=-\left\{\hat{\theta} r-\lim _{n \longrightarrow \infty} \frac{1}{n} \sup _{\boldsymbol{\alpha} \in S}\left[\log E_{P\left(R_{p, n}^{(\boldsymbol{\alpha})}(\omega)\right.}\left[\exp \left\{n \hat{\theta} R_{p, n}^{(\boldsymbol{\alpha})}(\omega)\right\}\right]\right]\right\}
$$

where $S$ is a compact set of feasible investments, $\hat{\theta}$ is in the neighbourhood of $\theta^{*}$, and $P\left(R_{p, n}^{(\boldsymbol{\alpha})}(\omega)\right)$ is the portfolio return distribution. Furthermore,

$$
\theta^{*}=\arg \lim _{n \longrightarrow \infty} \frac{1}{n} \inf _{\boldsymbol{\alpha} \in S} \sup _{\theta \in(0, \infty)}\left\{n \theta r-\log E_{P\left(R_{p, n}^{(\boldsymbol{\alpha})}(\omega)\right.}\left[\exp \left\{n \theta R_{p, n}^{(\boldsymbol{\alpha})}(\omega)\right\}\right]\right\}
$$

Similarly, we have

$$
\lim _{n \longrightarrow \infty} \frac{1}{n} \inf _{\boldsymbol{\alpha} \in S} \log \left(P\left(R_{p, n}^{(\boldsymbol{\alpha})}(\omega)<r\right)\right)=-\left\{\hat{\theta} r+\lim _{n \longrightarrow \infty} \frac{1}{n} \sup _{\boldsymbol{\alpha} \in S}\left[-\log E_{P\left(R_{p, n}^{(\boldsymbol{\alpha})}(\omega)\right.}\left[\exp \left\{n \hat{\theta} R_{p, n}^{(\boldsymbol{\alpha})}(\omega)\right\}\right]\right]\right\}
$$

where $\hat{\theta}$ is in the neighbourhood of $\theta^{*}$, where

$$
\theta^{*}=\arg \lim _{n \longrightarrow \infty} \frac{1}{n} \sup _{\substack{\theta \in(-\infty, 0) \\ \boldsymbol{\alpha} \in S}}\left\{n \theta r-\log E_{P\left(R_{p, n}^{(\boldsymbol{\alpha})}(\omega)\right)}\left[\exp \left\{n \theta R_{p, n}^{(\boldsymbol{\alpha})}(\omega)\right\}\right]\right\}
$$

Proof. See Appendix.

If the logarithmic expectation in the RHS of (12) or (15) is a concave function of $\boldsymbol{\alpha}$ in the compact set $S$, (12) and (13) imply that if the portfolio is large enough, either maximization of the overperformance probability or minimization of the underperformance probability is equivalent to maximization of an expected utility function. However, if the logarithmic expectation in (12) 
or (13) is a monotonic function, the solution is trivial since the optimal portfolio lies on the boundary of the investment set $S$.

As shown in Section 2.3.1, when a portfolio is very large, the number of individual central moments increases; the structure of dependence becomes rather complicated. This can hinder our analysis of the effects of individual skewnesses and correlations on optimal portfolio and asymmetric risk. For ease of exposition, we analyze the case when there are two risky assets. First, the case of two independent asset returns is analyzed.

\subsubsection{Assets are uncorrelated}

The mgf of $R_{p, 2}^{(\alpha)}(\omega)=\alpha \tilde{r}_{1}+(1-\alpha) \tilde{r}_{2}$, where $\tilde{r}_{i} \sim \operatorname{mixture}\left(p_{i}, \operatorname{Gamma}\left(\gamma_{i, 1}, \kappa_{i, 1}\right), \operatorname{Gamma}\left(\gamma_{i, 2}, \kappa_{i, 2}\right)\right)$ for $i=1,2$ as defined in Section 2.3.2, is given by

$$
\begin{aligned}
m g f(\theta, \alpha) & =p_{1} p_{2} \frac{1}{\left(1-\theta \alpha \gamma_{1,1}\right)^{\kappa_{1,1}}\left(1-\theta(1-\alpha) \gamma_{2,1}\right)^{\kappa_{2,1}}} \\
& +\left(1-p_{1}\right)\left(1-p_{2}\right) \frac{1}{\left(1+\theta \alpha \gamma_{1,2}\right)^{\kappa_{1,2}}\left(1+\theta(1-\alpha) \gamma_{2,2}\right)^{\kappa_{2,2}}} \\
& +p_{1}\left(1-p_{2}\right) \frac{1}{\left(1-\theta \alpha \gamma_{1,1}\right)^{\kappa_{1,1}}\left(1+\theta(1-\alpha) \gamma_{2,2}\right)^{\kappa_{2,2}}} \\
& +p_{2}\left(1-p_{1}\right) \frac{1}{\left(1+\theta \alpha \gamma_{2,1}\right)^{\kappa_{2,1}}\left(1-\theta(1-\alpha) \gamma_{1,2}\right)^{\kappa_{1,2}}}
\end{aligned}
$$

where the parameters $\left(\gamma_{i, 1}, \kappa_{i, 1}, \gamma_{i, 2}, \kappa_{i, 2}\right)$ for $i=1,2$ can be estimated from actual data by using the procedures described in Section 2.3.2.

To study the effects of the individual skewnesses or all the other high order moments of $R_{p, 2}^{(\alpha)}(\omega)$ on the optimal portfolio and the risk bound, we need to use some Edgeworth expansions of the mgf. The second order Edgeworth expansion (i.e., the mgf is truncated up to the second 
moment) is given by

$$
\begin{aligned}
\mathcal{E}_{2}(\theta, \alpha) & =\exp \left\{\theta E\left[R_{p, 2}^{(\alpha)}(\omega)\right]+\frac{1}{2} \theta^{2} E\left[R_{p, 2}^{(\alpha)}(\omega)-E\left[R_{p, 2}^{(\alpha)}(\omega)\right]\right]^{2}+\text { remainder }\right\} \\
& =\exp \left\{\theta\left(\alpha\left(p_{1} \gamma_{1,1} \kappa_{1,1}-\left(1-p_{1}\right) \gamma_{1,2} \kappa_{1,2}\right)+(1-\alpha)\left(p_{2} \gamma_{2,1} \kappa_{2,1}-\left(1-p_{2}\right) \gamma_{2,2} \kappa_{2,2}\right)\right)\right. \\
& +\frac{1}{2} \theta^{2}\left(\alpha\left(p_{1} \gamma_{1,1}^{2} \kappa_{1,1}+\left(1-p_{2}\right) \gamma_{2,2}^{2} \kappa_{2,2}\right)+(1-\alpha)\left(p_{2} \gamma_{2,1}^{2} \kappa_{2,1}+\left(1-p_{2}\right) \gamma_{2,2}^{2} \kappa_{2,2}\right)\right) \\
& + \text { remainder }\} .
\end{aligned}
$$

The third order Edgeworth expansion (i.e., the mgf is truncated up to the third moment) is given by

$$
\begin{aligned}
\mathcal{E}_{3}(\theta, \alpha) & =\exp \left\{\theta E\left[R_{p, 2}^{(\alpha)}(\omega)\right]+\frac{1}{2} \theta^{2} E\left[R_{p, 2}^{(\alpha)}(\omega)-E\left[R_{p, 2}^{(\alpha)}(\omega)\right]\right]^{2}+\frac{1}{6} \theta^{3} E\left[R_{p, 2}^{(\alpha)}(\omega)-E\left[R_{p, 2}^{(\alpha)}(\omega)\right]\right]^{3}\right. \\
& + \text { remainder }\} \\
& =\exp \left\{\theta\left(\alpha\left(p_{1} \gamma_{1,1} \kappa_{1,1}-\left(1-p_{1}\right) \gamma_{1,2} \kappa_{1,2}\right)+(1-\alpha)\left(p_{2} \gamma_{2,1} \kappa_{2,1}-\left(1-p_{2}\right) \gamma_{2,2} \kappa_{2,2}\right)\right)\right. \\
& +\frac{1}{2} \theta^{2}\left(\alpha\left(p_{1} \gamma_{1,1}^{2} \kappa_{1,1}+\left(1-p_{2}\right) \gamma_{2,2}^{2} \kappa_{2,2}\right)+(1-\alpha)\left(p_{2} \gamma_{2,1}^{2} \kappa_{2,1}+\left(1-p_{2}\right) \gamma_{2,2}^{2} \kappa_{2,2}\right)\right) \\
& +\frac{1}{3} \theta^{3}\left(\alpha\left(p_{1} \gamma_{1,1}^{3} \kappa_{1,1}-\left(1-p_{2}\right) \gamma_{2,2}^{3} \kappa_{2,2}\right)+(1-\alpha)\left(p_{2} \gamma_{2,1}^{3} \kappa_{2,1}-\left(1-p_{2}\right) \gamma_{2,2}^{3} \kappa_{2,2}\right)\right) \\
& + \text { remainder }\}
\end{aligned}
$$

As justified in (3), the underperformance probability is always bounded from above by $\exp \left\{-\Lambda_{2}(r, \theta, \alpha)\right\}$, where $\Lambda_{2}(r, \theta, \alpha)=r \theta-\log (m g f(\theta, \alpha)) .(m g f(\theta, \alpha)$ and its approximations are given in (14), (15), and (16) respectively.) Thus the upper bound is called the risk bound. To find an optimal portfolios for a given risk-aversion degree $\hat{\theta}$, we can use the Newton Raphson Algorithm to maximize $-m g f(\hat{\theta}, \alpha), \mathcal{E}_{2}(\hat{\theta}, \alpha)$, and $\mathcal{E}_{3}(\hat{\theta}, \alpha)$ respectively

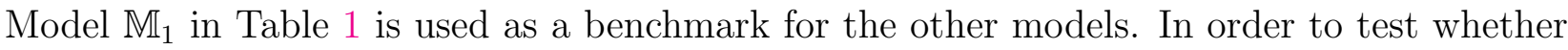
there is an effect of individual skewnesses or other high order moments on the optimal portfolio and the risk bound, we need to formulate a set of statistical hypotheses as follows:

\footnotetext{
${ }^{2}$ As shown in Burden and Fairs (1997), this algorithm has the degree of convergence $\mathbb{N}^{1 / 2}$, where $(\mathbb{N})$ is the number of iterations.
} 
Table 1: Model description

\begin{tabular}{ccc}
\hline Model & & The deviations function is based on \\
\cline { 1 - 1 } $\mathbb{M}_{1}$ & & $(15)$ \\
$\mathbb{M}_{2}$ & & $(16)$ \\
$\mathbb{M}_{3}$ &
\end{tabular}

1. The null hypothesis that there is a sole effect of the skewnesses on the optimal portfolio is

$$
\left.\mathbb{H}_{0}^{(1)}: \mid \hat{\alpha}_{\mathbb{M}_{2}} \hat{\theta}\right)-\hat{\alpha}_{\mathbb{M}_{1}}(\hat{\theta}) \mid \leq \epsilon
$$

where $\epsilon$ is an arbitrary small positive number, and $\hat{\theta}$ is a given risk aversion parameter.

2. The null hypothesis that there are effects of the skewnesses and all the other high order moments on the optimal portfolio is

$$
\mathbb{H}_{0}^{(2)}:\left|\hat{\alpha}_{\mathbb{M}_{3}}(\hat{\theta})-\hat{\alpha}_{\mathbb{M}_{1}}(\hat{\theta})\right| \leq \epsilon
$$

To test the null hypotheses $\mathbb{H}_{0}^{(1)}$ and $\mathbb{H}_{0}^{(2)}$, The one-cell contingency table test can be used. [See $\mathrm{Rad}$ (1973, page 393).] First, let us denote $(N)$ samples of the same size $(T)$ drawn from the true return distributions as $\left\{\left\{\left(\tilde{r}_{1 t}^{(i)}, \tilde{r}_{2 t}^{(i)}\right)\right\}_{t=1}^{T}\right\}_{i=1}^{N}$. However, we can split up a big sample of data in to $(N)$ small subsamples. Then, the $t$ test for the hypothesis $\mathbb{H}_{0}^{(1)}$ is given by

$$
t=\frac{o_{1}-e_{1}}{e_{1}}+\frac{o_{2}-e_{2}}{e_{2}} \sim \chi^{2}(1)
$$

where

$$
\begin{aligned}
o_{1} & =\#\left\{i \in[1, N]:\left|\hat{\alpha}_{\mathbb{M}_{2}, i}(\hat{\theta})-\hat{\alpha}_{\mathbb{M}_{1}, i}(\hat{\theta})\right| \leq \epsilon\right\} \\
o_{2} & =N-o_{1} \\
e_{1} & =0.95 N \\
e_{2} & =0.05 N
\end{aligned}
$$


where $\hat{\alpha}_{\mathbb{M}_{2}, i}(\hat{\theta})$ for $i=1, . ., N$ are the optimal portfolios estimated from the subsamples.

The $t$ test for the hypothesis $\mathbb{H}_{0}^{(2)}$ can also be formulated in a similar way. Furthermore, since skewness and asymmetry are typical features of asset returns, we believe that they have a strong effect on optimal portfolio, thus risk bound. This logic implies that the test for a sole effect of skewnesses on the optimal portfolio is equivalent to the test for asymmetry in the portfolio return distribution.

\subsubsection{Assets are correlated}

When asset returns are pairwise correlated, in view of Theorem 2 we need to specify a distribution for the portfolio return so that the mgf can be evaluated. An appropriate candidate is the asymmetric Gamma distribution as defined in Section 2.3.2. We apply the general theory given in Section 2.3.1 to construct parametrically optimal portfolios from actual return data.

Following Section 2.3.3, assuming that there are two risky assets. The portfolio return is $R_{p, 2}^{(\alpha)}(\omega)=\alpha \tilde{r}_{1}+(1-\alpha) \tilde{r}_{2}$, where $R_{p, 2}^{(\alpha)}(\omega) \sim \operatorname{mixture}\left(p(\alpha), \operatorname{Gamma}\left(\gamma_{1}(\alpha), \kappa_{1}(\alpha)\right), \operatorname{Gamma}\left(\gamma_{2}(\alpha), \kappa_{2}(\alpha)\right)\right)$. Then, we have two different cases.

- The Asymmetric Portfolio Return Distribution

As shown in Section 2.3.2, for a given investable proportion $(\alpha)$, the ML estimates $\left(\hat{\gamma}_{1}(\alpha), \hat{\kappa}_{1}(\alpha)\right.$, 
$\left.\hat{\gamma}_{2}(\alpha), \hat{\kappa}_{2}(\alpha), \hat{p}(\alpha)\right)$, which are the functions of $\alpha$, are the solutions to (2Q)-(24), i.e.,

$$
\begin{aligned}
\frac{\Gamma^{\prime}\left(\kappa_{1}(\alpha)\right)}{\Gamma\left(\kappa_{1}(\alpha)\right)}-\log \left(\kappa_{1}(\alpha)\right)= & \frac{\sum_{t=1}^{T} \log \left(R_{p, 2, t}^{(\alpha)}(\omega) I_{[0, \infty)}\left(R_{p, 2, t}^{(\alpha)}(\omega)\right)\right)}{\#\left\{t \in[1, T]: R_{p, 2, t}^{(\alpha)}(\omega) \geq 0\right\}} \\
& -\log \left(\frac{\sum_{t=1}^{T} R_{p, 2, t}^{(\alpha)}(\omega) I_{[0, \infty)}\left(R_{p, 2, t}^{(\alpha)}(\omega)\right)}{\#\left\{t \in[1, T]: R_{p, 2, t}^{(\alpha)}(\omega) \geq 0\right\}}\right), \\
\frac{\Gamma^{\prime}\left(\kappa_{2}(\alpha)\right)}{\Gamma\left(\kappa_{2}(\alpha)\right)}-\log \left(\kappa_{2}(\alpha)\right)= & \frac{\sum_{t=1}^{T} \log \left(-R_{p, 2, t}^{(\alpha)}(\omega) I_{(-\infty, 0)}\left(R_{p, 2, t}^{(\alpha)}(\omega)\right)\right)}{\#\left\{t \in[1, T]: R_{p, 2, t}^{(\alpha)}(\omega)<0\right\}} \\
- & \log \left(-\frac{\sum_{t=1}^{T} R_{p, 2, t}^{(\alpha)}(\omega) I_{(-\infty, 0)}\left(R_{p, 2, t}^{(\alpha)}(\omega)\right)}{\#\left\{t \in[1, T]: R_{p, 2, t}^{(\alpha)}(\omega)<0\right\}}\right), \\
\gamma_{1}(\alpha)= & \frac{\#\left\{t \in[1, T]: R_{p, 2, t}^{(\alpha)}(\omega) \geq 0\right\} \kappa_{1}(\alpha)}{\sum_{t=1}^{T} R_{p, 2, t}^{(\alpha)}(\omega) I_{[0, \infty)}\left(R_{p, 2, t}^{(\alpha)}(\omega)\right)} \\
\gamma_{2}(\alpha)= & -\frac{\sum_{t=1}^{T} R_{p, 2, t}^{(\alpha)}(\omega) I_{(-\infty, 0)}\left(R_{p, 2, t}^{(\alpha)}(\omega)\right)}{\#\left\{t \in[1, T]: R_{p, 2, t}^{(\alpha)}(\omega)<0\right\} \kappa_{2}(\alpha)}, \\
p(\alpha)= & \frac{\#\left\{t \in[1, T]: R_{p, 2, t}^{(\alpha)}(\omega) \geq 0\right\}}{T^{2}} .
\end{aligned}
$$

- Partially Symmetric Portfolio Return Distribution

Let $\gamma_{1}(\alpha)$ be equal to $\gamma_{2}(\alpha)$ (i.e., $\gamma(\alpha)=\gamma_{1}(\alpha)=\gamma_{2}(\alpha)$ ). Then the ML estimates 
$\left(\hat{\gamma}(\alpha), \hat{\kappa}_{1}(\alpha), \hat{\kappa}_{2}(\alpha), \hat{p}(\alpha)\right)$ are the solutions to (25)-(28), i.e.,

$$
\begin{aligned}
& \frac{\Gamma^{\prime}\left(\kappa_{1}(\alpha)\right)}{\Gamma\left(\kappa_{1}(\alpha)\right)}-\log \left(\#\left\{t \in[1, T]: R_{p, 2, t}^{(\alpha)}(\omega) \geq 0\right\} \kappa_{1}(\alpha)+\#\left\{t \in[1, T]: R_{p, 2, t}^{(\alpha)}(\omega)<0\right\} \kappa_{2}(\alpha)\right) \\
& =\frac{1}{\#\left\{t \in[1, T]: R_{p, 2, t}^{(\alpha)}(\omega) \geq 0\right\}} \sum_{t=1}^{T} \log \left(R_{p, 2, t}^{(\alpha)}(\omega) I_{[0, \infty)}\left(R_{p, 2, t}^{(\alpha)}(\omega)\right)\right) \\
& -\log \sum_{t=1}^{T} R_{p, 2, t}^{(\alpha)}(\omega)\left(I_{[0, \infty)}\left(R_{p, 2, t}^{(\alpha)}(\omega)\right)-I_{(-\infty, 0)}\left(R_{p, 2, t}^{(\alpha)}(\omega)\right)\right), \\
& \frac{\Gamma^{\prime}\left(\kappa_{2}(\alpha)\right)}{\Gamma\left(\kappa_{2}(\alpha)\right)}-\log \left(\#\left\{t \in[1, T]: R_{p, 2, t}^{(\alpha)}(\omega) \geq 0\right\} \kappa_{1}(\alpha)+\#\left\{t \in[1, T]: R_{p, 2, t}^{(\alpha)}(\omega)<0\right\} \kappa_{2}(\alpha)\right) \\
& =\frac{1}{\#\left\{t \in[1, T]: R_{p, 2, t}^{(\alpha)}(\omega)<0\right\}} \sum_{t=1}^{T} \log \left(-R_{p, 2, t}^{(\alpha)}(\omega) I_{(-\infty, 0)}\left(R_{p, 2, t}^{(\alpha)}(\omega)\right)\right) \\
& \text { - } \log \sum_{t=1}^{T} R_{p, 2, t}^{(\alpha)}(\omega)\left(I_{[0, \infty)}\left(R_{p, 2, t}^{(\alpha)}(\omega)\right)-I_{(-\infty, 0)}\left(R_{p, 2, t}^{(\alpha)}(\omega)\right)\right), \\
& \gamma(\theta)=\frac{\sum_{t=1}^{T} R_{p, 2, t}^{(\alpha)}(\omega)\left(I_{[0, \infty)}\left(R_{p, 2, t}^{(\alpha)}(\omega)\right)-I_{(-\infty, 0)}\left(R_{p, 2, t}^{(\alpha)}(\omega)\right)\right)}{\#\left\{t \in[1, T]: R_{p, 2, t}^{(\alpha)}(\omega) \geq 0\right\} \kappa_{1}(\alpha)+\#\left\{t \in[1, T]: R_{p, 2, t}^{(\alpha)}(\omega)<0\right\} \kappa_{2}(\alpha)}, \\
& p(\alpha)=\frac{\#\left\{t \in[1, T]: R_{p, n, t}^{(\alpha)}(\omega) \geq 0\right\}}{T} \text {. }
\end{aligned}
$$

Note that neither (20)-(24) nor (25)-(28) exist close form solutions. For brevity, we shall focus on (20)-(24); (25)-(28) can be handled in a similar way. An interpolation technique can be applied to estimate $\left(\hat{\gamma}_{1}(\alpha), \hat{\kappa}_{1}(\alpha), \hat{\gamma}_{2}(\alpha), \hat{\kappa}_{2}(\alpha), \hat{p}(\alpha)\right)$. Cubic splines (see e.g., Burden and Fairs, 1997) can be used to fit these relationships.

Let $\left(\operatorname{cs}_{1}(\alpha), \operatorname{cs}_{2}(\alpha), \operatorname{cs}_{3}(\alpha), \operatorname{cs}_{4}(\alpha), \operatorname{cs}_{5}(\alpha)\right)$ denote the fitted cubic splines of the ML estimates $\left(\hat{\gamma}_{1}(\alpha), \hat{\kappa}_{1}(\alpha), \hat{\gamma}_{2}(\alpha), \hat{\kappa}_{2}(\alpha), \hat{p}(\alpha)\right)$ resp. Then, the fitted mgf of $R_{p, 2}^{(\alpha)}(\omega)$ is given by

$$
\widehat{m g f}(\theta, \alpha)=\operatorname{cs}_{5}(\alpha)\left(\frac{1}{1-\theta \operatorname{cs}_{1}(\alpha)}\right)^{\operatorname{cs}_{2}(\alpha)}+\left(1-\operatorname{cs}_{5}(\alpha)\right)\left(\frac{1}{1+\theta \operatorname{cs}_{3}(\alpha)}\right)^{\operatorname{cs}_{4}(\alpha)}
$$

To study the effect of asymmetric dependence gauged by the nonlinear measures of correlation as defined in Section 2.2 on the optimal portfolio we need to examine the sensitivity of the portfolio skewness to changes in the nonlinear measures of correlation. Thus, some Edgeworth expansions 
of the fitted mgf can be used. The second order Edgeworth expansion is given by

$$
\begin{aligned}
\widehat{\mathcal{E}}_{2}(\theta, \alpha) & =\exp \left\{\theta\left(\operatorname{cs}_{5}(\alpha) \operatorname{cs}_{1}(\alpha) \operatorname{cs}_{2}(\alpha)-\left(1-\operatorname{cs}_{5}(\alpha)\right) \operatorname{cs}_{3}(\alpha) \operatorname{cs}_{4}(\alpha)\right)\right. \\
& \left.+\frac{1}{2} \theta^{2}\left(\operatorname{cs}_{5}(\alpha) \operatorname{cs}_{1}^{2}(\alpha) \operatorname{cs}_{2}(\alpha)+\left(1-\operatorname{cs}_{5}(\alpha)\right) \operatorname{cs}_{3}^{2}(\alpha) \operatorname{cs}_{4}(\alpha)\right)+\text { remainder }\right\}
\end{aligned}
$$

The third order Edgeworth expansion is given by

$$
\begin{aligned}
\widehat{\mathcal{E}}_{3}(\theta, \alpha) & =\exp \left\{\theta\left(\operatorname{cs}_{5}(\alpha) \operatorname{cs}_{1}(\alpha) \operatorname{cs}_{2}(\alpha)-\left(1-\operatorname{cs}_{5}(\alpha)\right) \operatorname{cs}_{3}(\alpha) \operatorname{cs}_{4}(\alpha)\right)\right. \\
& +\frac{1}{2} \theta^{2}\left(\operatorname{cs}_{5}(\alpha) \operatorname{cs}_{1}^{2}(\alpha) \operatorname{cs}_{2}(\alpha)+\left(1-\operatorname{cs}_{5}(\alpha)\right) \operatorname{cs}_{3}^{2}(\alpha) \operatorname{cs}_{4}(\alpha)\right) \\
& \left.+\frac{1}{3} \theta^{3}\left(\operatorname{cs}_{5}(\alpha) \operatorname{cs}_{1}^{3}(\alpha) \operatorname{cs}_{2}(\alpha)-\left(1-\operatorname{cs}_{5}(\alpha)\right) \operatorname{cs}_{3}^{3}(\alpha) \operatorname{cs}_{4}(\alpha)\right)+\text { remainder }\right\}
\end{aligned}
$$

As shown in Theorem 20, the optimal portfolios $(\hat{\alpha}(\hat{\theta}))$ are the maximal values of $-\widehat{m g} f(\hat{\theta}, \alpha)$, $-\widehat{\mathcal{E}}_{2}(\hat{\theta}, \alpha)$, and $-\widehat{\mathcal{E}}_{3}(\hat{\theta}, \alpha)$ resp., where $\hat{\theta}$ is in the neighbourhood of $\theta^{*}$ as defined in Theorem 2. The models are summarized in Table 2. For the (partially) symmetric case as defined by

Table 2: Model descriptions

\begin{tabular}{ccc}
\hline Model & & The deviations function is based on \\
\cline { 1 - 1 } $\mathbb{M}_{4}$ & & $(30)$ \\
$\mathbb{M}_{5}$ & & $(31)$ \\
$\mathbb{M}_{6}$ & $(29)$ \\
\hline
\end{tabular}

(25)-(28), we can define the models $\mathbb{M}_{7}$ (the benchmark model), $\mathbb{M}_{8}$ (the third order Edgeworth expansion), and $\mathbb{M}_{9}$ (the exact moment) in a similar way.

As pointed out in (8), although high order measures of correlations can not be recovered from the central moments of the optimal portfolio return, their pairwise linear relationships can be recovered, i.e.,

$$
\hat{\lambda}_{11}=\frac{E\left[R_{p, 2}^{(\hat{\alpha})}(\omega)-E\left[R_{p, 2}^{(\hat{\alpha})}(\omega)\right]\right]^{2}-\hat{\alpha}^{2} E\left[\tilde{r}_{1}-E\left[\tilde{r}_{1}\right]\right]^{2}-(1-\hat{\alpha})^{2} E\left[\tilde{r}_{2}-E\left[\tilde{r}_{2}\right]\right]^{2}}{\sqrt{E\left[\tilde{r}_{1}-E\left[\tilde{r}_{1}\right]\right]^{2} E\left[\tilde{r}_{2}-E\left[\tilde{r}_{2}\right]\right]^{2}}},
$$




$$
\begin{aligned}
& \hat{\lambda}_{21} \hat{\alpha}^{2}(1-\hat{\alpha}) \sqrt{E\left[\tilde{r}_{1}-E\left[\tilde{r}_{1}\right]\right]^{4} E\left[\tilde{r}_{2}-E\left[\tilde{r}_{2}\right]\right]^{2}} \\
& \quad+\hat{\lambda}_{12} \hat{\alpha}(1-\hat{\alpha})^{2} \sqrt{E\left[\tilde{r}_{1}-E\left[\tilde{r}_{1}\right]\right]^{2} E\left[\tilde{r}_{2}-E\left[\tilde{r}_{2}\right]\right]^{4}} \\
& =\frac{2}{3 \sqrt{6}}\left(E\left[R_{p, 2}^{(\hat{\alpha})}(\omega)-E\left[R_{p, 2}^{(\hat{\alpha})}(\omega)\right]\right]^{3}-\hat{\alpha}^{3} E\left[\tilde{r}_{1}-E\left[\tilde{r}_{1}\right]\right]^{3}-(1-\hat{\alpha})^{3} E\left[\tilde{r}_{2}-E\left[\tilde{r}_{2}\right]\right]^{3}\right),
\end{aligned}
$$

where

$$
\begin{aligned}
E\left[R_{p, 2}^{(\hat{\alpha})}(\omega)-E\left[R_{p, 2}^{(\hat{\alpha})}(\omega)\right]\right]^{2} & =\operatorname{cs}_{5}(\hat{\alpha}) \operatorname{cs}_{1}^{2}(\hat{\alpha}) \operatorname{cs}_{2}(\hat{\alpha})+\left(1-\operatorname{cs}_{5}(\hat{\alpha})\right) \operatorname{cs}_{3}^{2}(\hat{\alpha}) \operatorname{cs}_{4}(\hat{\alpha}), \\
E\left[R_{p, 2}^{(\hat{\alpha})}(\omega)-E\left[R_{p, 2}^{(\hat{\alpha})}(\omega)\right]\right]^{3} & =\operatorname{cs}_{5}(\hat{\alpha}) \operatorname{cs}_{1}^{3}(\hat{\alpha}) \operatorname{cs}_{3}(\hat{\alpha})-\left(1-\operatorname{cs}_{5}(\hat{\alpha})\right) \operatorname{cs}_{3}^{3}(\hat{\alpha}) \operatorname{cs}_{4}(\hat{\alpha}), \\
E\left[\tilde{r}_{1}-E\left[\tilde{r}_{1}\right]\right]^{2} & =\hat{p}_{1} \hat{\gamma}_{1,1}^{2} \hat{\kappa}_{1,1}+\left(1-\hat{p}_{1}\right) \hat{\gamma}_{1,2}^{2} \hat{\kappa}_{1,2}, \\
E\left[\tilde{r}_{1}-E\left[\tilde{r}_{1}\right]\right]^{3} & =\hat{p}_{1} \hat{\gamma}_{1,1}^{3} \hat{\kappa}_{1,1}-\left(1-\hat{p}_{1}\right) \hat{\gamma}_{1,2}^{3} \hat{\kappa}_{1,2}, \\
E\left[\tilde{r}_{1}-E\left[\tilde{r}_{1}\right]\right]^{4} & =\hat{p}_{1} \hat{\gamma}_{1,1}^{4} \hat{\kappa}_{1,1}+\left(1-\hat{p}_{1}\right) \hat{\gamma}_{1,2}^{4} \hat{\kappa}_{1,2}, \\
E\left[\tilde{r}_{2}-E\left[\tilde{r}_{2}\right]\right]^{2} & =\hat{p}_{2} \hat{\gamma}_{2,1}^{2} \hat{\kappa}_{2,1}+\left(1-\hat{p}_{2}\right) \hat{\gamma}_{2,2}^{2} \hat{\kappa}_{2,2}, \\
E\left[\tilde{r}_{2}-E\left[\tilde{r}_{2}\right]\right]^{3} & =\hat{p}_{2} \hat{\gamma}_{2,1}^{3} \hat{\kappa}_{2,1}-\left(1-\hat{p}_{2}\right) \hat{\gamma}_{2,2}^{3} \hat{\kappa}_{2,2}, \\
E\left[\tilde{r}_{2}-E\left[\tilde{r}_{2}\right]\right]^{4} & =\hat{p}_{2} \hat{\gamma}_{2,1}^{4} \hat{\kappa}_{2,1}+\left(1-\hat{p}_{2}\right) \hat{\gamma}_{2,2}^{4} \hat{\kappa}_{2,2},
\end{aligned}
$$

and $\hat{\lambda}_{11}$ is the recovered linear correlation. $\hat{\lambda}_{12}$ and $\hat{\lambda}_{21}$ are the recovered first nonlinear correlation and the recovered second one as given in Section 2.2. $\hat{\alpha}$ is the optimal portfolio estimated from the models in Table 2. The other parameters with hats $\left({ }^{\wedge}\right)$ are the ML estimates of the asymmetric Gamma distributions as defined in Section 2.3.2.

(32) and (B3) shows the precise relationship between the optimal portfolio $(\hat{\alpha}(\hat{\theta}))$ and the recovered linear correlation $\left(\hat{\lambda}_{11}\right)$ or the recovered nonlinear correlations $\left(\hat{\lambda}_{12}, \hat{\lambda}_{21}\right)$. The effect of the measures of correlation on the optimal portfolio is rather sensitive to the interpolation polynomials $\mathrm{cs}_{1}, \mathrm{cs}_{2}, \mathrm{cs}_{3}, \mathrm{cs}_{4}$ and $\mathrm{cs}_{5}$. Furthermore, (32) implies that changes in the individual variances and in the linear correlation may cancel out one other so that the effect of the linear correlation on the optimal portfolio becomes negligible. (33) implies that changes in the nonlinear correlations ceteris paribus may cancel out one other so that the optimal portfolio remains intact. Hence, there exists a configurational possibility of the linear correlation or the nonlinear 
correlations such that the total impact of asymmetric dependence on the optimal portfolio is negligible. We will address this point further in Section 0 .

Let $\mathbb{M}_{4}$ denotes the benchmark model. In order to test whether there is an effect of the second order nonlinear correlations or the other high order nonlinear correlations on the optimal portfolio, we need to formulate a set of statistical hypotheses as follows:

1. The null hypothesis that there is a sole effect of the second order nonlinear correlations on the optimal portfolio is

$$
\mathbb{H}_{0}^{(3)}:\left|\hat{\alpha}_{\mathbb{M}_{5}}(\hat{\theta})-\hat{\alpha}_{\mathbb{M}_{4}}(\hat{\theta})\right| \leq \epsilon
$$

where $\epsilon$ is an arbitrary small number. $\hat{\theta}$ is in the neighbourhood of $\theta^{*}$ as defined in Theorem 2.

2. The null hypothesis that there is an effect of the other high order nonlinear correlations on the optimal portfolio is

$$
\mathbb{H}_{0}^{(4)}:\left|\hat{\alpha}_{\mathbb{M}_{6}}(\hat{\theta})-\hat{\alpha}_{\mathbb{M}_{4}}(\hat{\theta})\right| \leq \epsilon
$$

where $\hat{\alpha}_{\mathbb{M}_{4}}, \hat{\alpha}_{\mathbb{M}_{5}}$, and $\hat{\alpha}_{\mathbb{M}_{6}}$ are the optimal portfolios estimated from the models in Table 2 .

The null hypotheses for the partially symmetric case (i.e., the models $\mathbb{M}_{7}-\mathbb{M}_{9}$ ) can be formulated similarly. The one-cell contingency table test as proposed in Section 2.3.3 can be used to test $\mathbb{H}_{0}^{(3)}$ and $\mathbb{H}_{0}^{(4)}$. However, since asymmetric dependence is a typical phenomenon in the market, thus it has an evident impact on optimal asset allocation (see e.g. Patton, 2004). We will confirm that there is a strong effect of the second order nonlinear correlations on the optimal portfolio by using some empirical evidences.

\section{EMPIRICAL EVIDENCE}

In this section, we provide some short simulations to illustrate the portfolio optimization technique proposed in Section 2. By using equity data Bangkok SET index[3 and S\&P 500, we also investigate empirically the effect of individual skewnesses and nonlinear correlations on optimal

\footnotetext{
${ }^{3}$ Source: www.globaldata.com. Bangkok SET index is the FX adjusted equity price denominated in USD.
} 
portfolio and risk bound.

\section{Data description}

Data of Bangkok SET and S\&P 500 are available monthly from 1975 to 2001. Arithmetic

return is calculated by $\tilde{r}_{t}=\frac{P_{t}-P_{t-1}}{P_{t-1}}$. The sample statistics of the returns are reported in Table 3. The kernel return densities are plotted in Figure 1. The mean and the variance of S\&P 500 are higher while the kurtosises are roughly equal. However, Bangkok SET has a positive skewness while S\&P 500 has a negative one. The first sample nonlinear correlation and the second one, that are apparently unequal, indicate a strong asymmetric dependence between the two assets. Hence, in view of the example given in Section 2.2, this case is indeed interesting to investigate.

The ML estimates of the parameters of the return distributions for Bangkok SET and S\&P 500 are reported in Table 1 . The same phenomenon as shown in Table 3 can be noticed. That is, the mean and the standard deviation of S\&P 500 are higher than those of Bangkok SET as shown in the second panel of Table 6 . The skewness of S\&P 500 is negative while it is positive for Bangkok SET. Moreover, the standard errors of the ML estimates in the (partially) symmetric case are in general smaller than those in the asymmetric case. This indicates that the asset returns are better modelled with a partially symmetric Gamma distribution. The type of symmetry can be further confirmed by the LR test as given in Table 日.

First, we choose a benchmark return $(r)$ which must be less than the mean of the portfolio return (see Remark 2.1). Next, we estimate the optimal portfolios and the risk bounds that are the upper bounds of $P\left\{R_{p, 2}^{(\alpha)}(\omega)<r\right\}$ for the models in Tables 1 and 2 . Finally, we investigate the effect of the degree of absolute risk aversion, individual skewnesses and the nonlinear correlations on the optimal portfolio by examining the portfolio mean, the portfolio standard deviation, the portfolio skewness and the deviations function.

\section{Asymmetric Gamma distributions, optimal portfolios, and their risk bounds}


First, we shall assume that the asset returns are uncorrelated. The benchmark is set to -1\%. Table $\mathrm{G}$ indicates that for three models $\mathbb{M}_{1}, \mathbb{M}_{2}$, and $\mathbb{M}_{3}$, the proportion held in S\&P 500 decreases as the degree of absolute risk aversion increases. Figure - shows that the growth rate (i.e., the deviations function) curve tends to shift upward as the degree of risk aversion increases. This implies that the risk bound decreases as an investor becomes more averse to the likelihood that the portfolio return underperforms a benchmark.

As shown in Table 7 , since $\mathbb{M}_{1}$ is the benchmark model, the pro of including the skewnesses is evident since the mean, the standard deviation, and the skewness of the optimal portfolio return in Model $\mathbb{M}_{2}$ are higher than those in Model $\mathbb{M}_{1}$. This is because the deviations function approximated with the third order Edgeworth expansion supports low standard deviation and positive portfolio skewness. However, in Model $\mathbb{M}_{3}$ where the exact mgf is used, the mean, the variance, and the skewness of the optimal portfolio return are less than those in Model $\mathbb{M}_{1}$. This is due to the presence of the high order moments which may dominate the low order moments, thus diminish the impact of the skewnesses. As a result, the high order moments decrease the mean, the variance, and the skewness of the optimal portfolio return whilst the individual skewnesses solely increase those of the optimal portfolio return. Hence, a rational investor prefers Model $\mathbb{M}_{2}$ to the other two models since this model gives a higher mean and a higher skewness at the cost of a slightly higher standard deviation.

Next, we assume that the asset returns are correlated. In this case, we need to make assumptions about the marginal return distributions and the portfolio return distribution. Suppose that the portfolio return distribution is an asymmetric Gamma distribution. The method proposed in Section 2.3 does not require us to estimate the measures of nonlinear correlation explicitly in order to estimate the optimal portfolio. Instead, the optimal portfolio is found by simultaneous maximization of the deviations function and the average log likelihood function.

In Table 6, first note that the optimal proportions held in $\mathrm{S} \& \mathrm{P} 500$ in $\mathbb{M}_{4}-\mathbb{M}_{9}$ are much less 
than those in $\mathbb{M}_{1}-\mathbb{M}_{3}$ where asset returns are assumed to be uncorrelated. Thus, correlation apparently has a rather significant impact on optimal portfolio. By setting the benchmark return to $-1 \%$ which is less than the sample mean of an asset return, we can observe that the optimal portfolio initially increases then decreases at the point where the degree of absolute risk aversion $\left(\theta^{*}\right)$ is 14.5 obtained by maximizing the deviations function w.r.t. both $\alpha$ and $\theta$. As seen in Figures 6 and 0 , the growth rate (the deviations function) curve for $\theta=14.5$ is on the top of the other curves. In another word, the optimal portfolio, that is a function of the degree of risk aversion, has smile shape. Furthermore, the risk bounds are much less than those in $\mathbb{M}_{1}-\mathbb{M}_{3}$. Thus, the models $\mathbb{M}_{4}-\mathbb{M}_{9}$ are much less risky than the models $\mathbb{M}_{1}-\mathbb{M}_{3}$. This is a benefit of asymmetric dependence in the portfolio analysis.

In addition, the effect of the nonlinear correlations on the optimal portfolio can be examined in Table 0. In the asymmetric case, $\mathbb{M}_{4}$ is the benchmark model, $\mathbb{M}_{5}$, that is obtained by an application of the third order Edgeworth expansion of the exact mgf, yields a higher mean and a lower standard deviation at the cost of a lower skewness. Contrary to Models $\mathbb{M}_{1}-\mathbb{M}_{3}$, the presence of the second order nonlinear correlations as defined in Section 2.2 reduces the portfolio skewness whilst increasing the portfolio mean. In the symmetric case, $\mathbb{M}_{7}$ is the benchmark model, $\mathbb{M}_{8}$ yields a higher mean, lower variance, and a higher skewness. An interesting point to note is that the presence of the nonlinear correlations increases not only the portfolio skewness but also the portfolio mean. Since investors always prefer skewness, Model $\mathbb{M}_{8}$ is obviously preferable to Model $\mathbb{M}_{7}$. However, Model $\mathbb{M}_{9}$, which uses the exact mgf, is the most favourable model since it gives a higher mean, a lower standard deviation, and a higher skewness. This is due to the fact that the high order correlations that combine with the second order nonlinear correlations support the deviations function to effectively generate a high mean, a low standard deviation, and a high skewness. Hence, the investment efficiency can be enhanced by incorporating high order correlations.

Alternatively, we can use a simulation approach to investigate separately the effect of the linear correlation $\left(\lambda_{11}\right)$ and a combination of the nonlinear correlations $\left(\lambda_{12}\right)$ and $\left(\lambda_{21}\right)$ on 
the optimal portfolio. First, we generate 2000 bivariate samples of two Gamma random variables with the true parameters as given in Table 1 . Next, we use Model $\mathbb{M}_{6}$ or $\mathbb{M}_{9}$ to estimate an optimal portfolio by total maximization of the deviations function. The estimated optimal portfolio is then used to recover the linear correlation and nonlinear ones from each bivariate sample. Eventually, we have a sample of 2000 optimal portfolios together with recovered measures of correlation. As shown in Figure 3 , as the linear correlation increases, the proportion held in S\&P 500 noticeably decreases. This is compatible with the results in Table 6 that when the asset returns are correlated, the proportion invested in S\&P 500 decreases significantly. Now, we move on to examine Figure 4 . When Model $\mathbb{M}_{6}$ is used, the nonlinear correlations $\left(\lambda_{12}\right)$ and $\left(\lambda_{21}\right)$, that are of different signs, have no effect on the optimal portfolio since they can be cancelled out, while the ones, that are of the same signs, have a rather strong effect as shown in Figure 5(a). However, when Model $\mathbb{M}_{9}$ is used, regardless of the signs, the nonlinear correlations always have a strong effect on the optimal portfolio as seen in Figure 5(b). Thus, no effect of signs can be observed. This is due to the partial symmetry assumed for the portfolio return distribution. Hence, the sign effects of the nonlinear correlations are always stronger in the asymmetric case than in the partially symmetric case.

Therefore, the empirical results given in this section confirm our conjecture that asymmetric dependence between individual assets and asymmetry in the portfolio return are two typical types of asymmetry that have strong effects on optimal asset allocations. Moreover, we empirically show that highly efficient investment strategies can be devised by taking high order moments and high order correlations in to account. However, a general theory which can quantify the magnitude of effects is complicated to derive in our framework. This is an interesting topic for the future research.

\section{CONCLUSION}

In this paper, we apply the large deviations theory in the problem of selecting optimal investment for a large portfolio such that both criteria of minimizing the underperformance probability and 
maximizing the expected exponential utility function are satisfied. Then, we establish a theoretical framework for finding optimal portfolios when there are many risky assets. However, because analysis of the effect of correlation on optimal portfolio is rather complicated for a large number of assets, we shall focus on the special case of two assets. An asymmetric Gamma distribution is used to capture two types of asymmetry; 1) asymmetry in the portfolio return distribution 2) asymmetric dependence (gauged by the second order measures of nonlinear correlation) between risky assets. Empirical evidences provided in Section 3 confirm that there is a strong effect of asymmetry and nonlinear correlations on optimal portfolio.

However, since the analysis in this paper is based on the asymmetric Gamma distribution, our future research will focus on more realistic models such as; 1) the joint distribution of asset returns is a generalized mixture of Gamma distributions, 2) asset returns may depend on another economic indicator such as interest rate, yield spread, and market index etc., 3) The benchmark return can be stochastic. All the above extensions can be difficult problems. 


\section{Appendix A. Proofs of results}

The proof of Theorem 1 basically follows from the proof of Theorem 3 .

Theorem 3. Let us define ( $n$ ) IID random variables $\tilde{r}_{i}$ with the $m g f m g f(\theta)$, where $i=1, . ., n$. The upper tail probability of $\bar{r}_{n}=\frac{1}{n} \sum_{i=1}^{n} \tilde{r}_{i}$ is given by (A.36) or (A.37). That is,

$$
P\left\{\bar{r}_{n}>r\right\} \simeq \frac{1}{\sqrt{2 \pi n} \theta(r) \sigma(r)} \exp \{-n \Lambda(r)\}
$$

where $\sigma^{2}(r)=\frac{1}{\theta^{\prime}(r)}\left(=\frac{1}{\Lambda^{\prime \prime}(r)}\right), \Lambda(r)=\sup _{\theta>0}\left\{\theta r-\log E\left[\exp \left\{\theta \tilde{r}_{1}\right\}\right]\right\}$, and $\theta(r)=\arg \sup _{\theta>0}\{\theta r-$ $\left.\log E\left[\exp \left\{\theta \tilde{r}_{1}\right\}\right]\right\}$.

$$
P\left\{\bar{r}_{n}>r\right\}=\exp \{-n \theta(r)\} \exp \left\{\frac{1}{2} n \sigma^{4}(r) \theta^{2}(r)\right\}\left\{1-m g f\left(\sqrt{n} \sigma^{2}(r) \theta(r)\right)\right\}\left(1+o_{p}(1)\right) .
$$

Proof. See Zhulenev (1997).

Remark 4.1. Let $\theta_{+}=\sup \{\theta \in(0, \infty): \operatorname{mgf}(\theta)<\infty\}$ and $r_{+}=\lim _{\theta \longrightarrow \theta_{+}}\left\{\frac{m g f^{\prime}(\theta)}{m g f(\theta)}\right\}$. Since $\Lambda^{\prime}(r)=\theta(r)+\theta^{\prime}(r)\left\{r-\frac{m g f^{\prime}(\theta(r))}{m g f(\theta(r))}\right\}$. If $r=E\left[\tilde{r}_{1}\right]$ then $\theta\left(E\left[\tilde{r}_{1}\right]\right)=0$ since $\log (m g f(\theta))$ is a strictly convex function (i.e., $\Lambda(r)$ is a strictly concave function.) Therefore, for any $r \in\left(E\left[\tilde{r}_{1}\right], r_{+}\right)$, the maximal value of $\Lambda(r)$ is attained at a unique point $\theta(r)$, where the tangent line to $m g f(\theta(r))$ is parallel with the line $r \theta$. Moreover, the function $\theta(r)$ increases $\forall r \in\left(E\left[\tilde{r}_{1}\right], r_{+}\right)$. Further results can found in Zhulene? (1997).

Proof of Theorem 1. Now, we give a proof for the upper tail. The proof of the large deviations result for the lower tail is quite similar.

The portfolio return is given by

$$
R_{p, n}^{(\boldsymbol{\alpha})}(\omega)=\sum_{i=1}^{n} \alpha_{i} \tilde{r}_{i}
$$

where $\tilde{r}_{i} \sim \operatorname{IID}\left(\mu, \sigma^{2}\right)$. 
An application of Cramer's transformation yields

$$
P\left\{R_{p, n}^{(\boldsymbol{\alpha})}(\omega)>r\right\}=m g f(\theta(r)) \int_{r}^{\infty} \exp \{-\theta(r) u\} P\left\{R_{p, n}^{(\boldsymbol{\alpha}, r)}(\omega) \in d u\right\}
$$

where $R_{p, n}^{(\boldsymbol{\alpha}, r)}(\omega)$ is a weighted sum as (A.38) with the mean $(r)$ and the variance $\left(\sigma^{2}(r)\right) \cdot \operatorname{mg} f(\theta)$ is the mgf of $R_{p, n}^{(\boldsymbol{\alpha})}(\omega)$ and $\theta(r)=\operatorname{argsup}_{\theta>0}\{\theta r-\log m g f(\theta)\}$. [Note that since $\left.\frac{m g f^{\prime}(\theta)}{m g f(\theta)}\right|_{\theta=0}=\mu$, thus if $r=\mu$ then $\theta(r)=0$.] Hence, we have

$$
\begin{aligned}
& P\left\{R_{p, n}^{(\boldsymbol{\alpha})}(\omega)>r\right\}=\operatorname{mgf}(\theta(r)) \int_{0}^{\infty} \exp \{-\theta(r)(u+r)\} P\left\{R_{p, n}^{(\boldsymbol{\alpha}, r)}(\omega) \in r+d u\right\} \\
& =m g f(\theta(r)) \exp \{-r \theta(r)\} \int_{0}^{\infty} \exp \{-\theta(r) u\} P\left\{\frac{R_{p, n}^{(\boldsymbol{\alpha}, r)}(\omega)-r}{\sqrt{\sum_{i=1}^{n} \alpha_{i}^{2}} \sigma(r)} \in \frac{d u}{\sqrt{\sum_{i=1}^{n} \alpha_{i}^{2}} \sigma(r)}\right\} \\
& =\exp \{-(\theta(r) r-\log m g f(\theta(r)))\}
\end{aligned}
$$

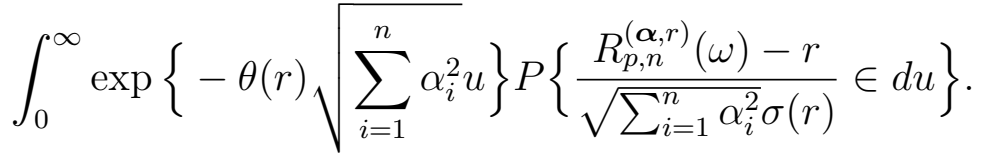

Applying the integral separation formula for $F^{(r)}(u)=P\left\{\frac{R_{p, n}^{(\boldsymbol{\alpha}, r)}(\omega)-r}{\sqrt{\sum_{i=1}^{n} \alpha_{i}^{2}} \sigma(r)} \leq u\right\}$, we have

$$
\begin{gathered}
P\left\{R_{p, n}^{(\boldsymbol{\alpha})}(\omega)>r\right\}=\theta(r) \sigma(r) \sqrt{\sum_{i=1}^{n} \alpha_{i}^{2}} \exp \{-(\theta(r) r-\log m g f(\theta(r)))\} \\
\int_{0}^{\infty} \exp \left\{\theta(r) \sigma(r) \sqrt{\left.\sum_{i=1}^{n} \alpha_{i}^{2} u\right\}}\left(F^{(r)}(u)-F^{(r)}(0)\right) d u .\right.
\end{gathered}
$$

Moreover, an Edgeworth expansion of $d F^{(r)}(u)$ yields

$$
\begin{aligned}
d F^{(r)}(u) & =P\left\{\frac{R_{p, n}^{(\boldsymbol{\alpha}, r)}(\omega)-r}{\sqrt{\sum_{i=1}^{n} \alpha_{i}^{2}} \sigma(r)}=u\right\} \\
& =\phi(u)-\frac{E\left[R_{p, n}^{(\boldsymbol{\alpha}, r)}(\omega)-r\right]^{3}}{\left(E\left[R_{p, n}^{(\boldsymbol{\alpha}, r)}(\omega)-r\right]^{2}\right)^{\frac{3}{2}}} \phi^{\prime \prime \prime}(u)+\text { remainder }
\end{aligned}
$$

where $\phi^{\prime \prime \prime}(u)$ is the third order derivative of the standard normal density $\phi(u)$. By substituting 
the approximation of $d F^{(r)}(u)$ in to $(\mathrm{A.40})$, we have

$$
\begin{aligned}
P\left\{R_{p, n}^{(\boldsymbol{\alpha})}(\omega)>r\right\} & =\beta(r) \exp \{-(\theta(r) r-\log \operatorname{mgf}(\theta(r)))\}\left\{\int_{0}^{\infty} \exp \{\beta(r) u\}(\Phi(u)-\Phi(0)) d u\right. \\
& \left.-\frac{E\left[R_{p, n}^{(\boldsymbol{\alpha}, r)}(\omega)-r\right]^{3}}{\left(E\left[R_{p, n}^{(\boldsymbol{\alpha}, r)}(\omega)-r\right]^{2}\right)^{\frac{3}{2}}} \int_{0}^{\infty} \exp \{\beta(r) u\}\left(\Phi^{(3)}(u)-\Phi^{(3)}(0)\right) d u\right\}+ \text { remainder }
\end{aligned}
$$

where $\Phi(u)$ is the lower tail of the standard normal distribution, $\Phi^{(3)}(u)$ is the lower tail of $\phi^{\prime \prime \prime}(u)$, and $\beta(r)=\theta(r) \sigma(r) \sqrt{\sum_{i=1}^{n} \alpha_{i}^{2}}$.

In view of

$$
\int_{0}^{\infty} \exp \{\theta u\} \exp \left\{-\frac{u^{2}}{2}\right\} d u=\sqrt{2 \pi} \exp \left\{\frac{\theta^{2}}{2}\right\}(1-\Phi(\theta))
$$

we can easily derive

$$
\begin{aligned}
\int_{0}^{\infty} \exp \{\beta(r) u\}(\Phi(u)-\Phi(0)) d u & =\frac{1}{\beta(r)}\left(\exp \left\{\frac{\beta^{2}(r)}{2}\right\}(1-\Phi(\beta(r)))\right) \\
\int_{0}^{\infty} \exp \{\beta(r) u\}\left(\Phi^{(3)}(u)-\Phi^{(3)}(0)\right) d u & =\exp \left\{\frac{\beta^{2}(r)}{2}\right\}(1-\Phi(\beta(r)))\left(\beta^{2}(r)+2\right) \\
& +\frac{1}{\sqrt{2 \pi}}\left(\beta(r)+\frac{1}{\beta(r)}\right) .
\end{aligned}
$$

Hence, we obtain

$$
\begin{aligned}
P\left\{R_{p, n}^{(\boldsymbol{\alpha})}(\omega)>r\right\} & =\beta(r) \exp \{-\Lambda(r)\}\left\{\frac{\exp \left\{\frac{\beta^{2}(r)}{2}\right\}[1-\Phi(\beta(r))]}{\beta(r)}\right. \\
& -\frac{E\left[R_{p, n}^{(\boldsymbol{\alpha}, r)}(\omega)-r\right]^{3}}{\left[E\left[R_{p, n}^{(\boldsymbol{\alpha}, r)}(\omega)-r\right]^{2}\right]^{\frac{3}{2}}}\left[\exp \left\{\frac{\beta^{2}(r)}{2}\right\}[1-\Phi(\beta(r))]\left[\beta^{2}(r)+2\right]\right. \\
& \left.\left.+\frac{1}{\sqrt{2 \pi}}\left(\beta(r)+\frac{1}{\beta(r)}\right)\right]+ \text { remainder }\right\}
\end{aligned}
$$

where $\Lambda(r)=\theta(r) r-\log m g f(\theta(r))$.

Since $\left(\sum_{i=1}^{n} \alpha_{i}^{2}\right)^{\frac{3}{2}} \geq \sum_{i=1}^{n} \alpha_{i}^{3}$ then $\sum_{i=1}^{n} \alpha_{i}^{3}$ approaches to zero faster than $\left(\sum_{i=1}^{n} \alpha_{i}^{2}\right)^{\frac{3}{2}}$ as $(n)$ is sufficiently large. Furthermore, since all the central moments of $\tilde{r}_{1}$ are finite, it follows that $\frac{E\left[R_{p, n}^{(\boldsymbol{\alpha}, r)}(\omega)-r\right]^{3}}{\left[E\left[R_{p, n}^{(\boldsymbol{\alpha}, r)}(\omega)-r\right]^{2}\right]^{\frac{3}{2}}}$ is negligible. The same argument can be applied to show that the remainder of 
(A.41) is negligible. Therefore, (A.41) can be written as

$$
P\left\{R_{p, n}^{(\boldsymbol{\alpha})}(\omega) \geq r\right\} \approx \exp \{-\Lambda(r)\} \exp \left\{\frac{\beta^{2}(r)}{2}\right\}[1-\Phi(\beta(r))]+o(1)
$$

Furthermore, by the approximation

$$
1-\Phi(\beta(r))=\frac{\exp \left\{-\frac{\beta^{2}(r)}{2}\right\}}{\sqrt{2 \pi \beta(r)}}
$$

we obtain the main result of Theorem 1 .

Finally, in order to show that $\sigma^{2}(r)^{2}=\frac{1}{\Lambda^{N}(r)}$ we make use of the following identity:

$$
\begin{aligned}
\Lambda^{\prime}(r) & =\theta(r), \\
\left.\frac{m g f^{\prime}(\theta)}{m g f(\theta)}\right|_{\theta=\theta(r)} & =r .
\end{aligned}
$$

Take the first derivative of A.42) w.r.t. $(r)$, we have

$$
\begin{aligned}
& \frac{\left[m g f^{\prime \prime}(\theta(r)) m g f(\theta(r))-\left(m g f^{\prime}(\theta(r))\right)^{2}\right] \theta^{\prime}(r)}{m g f^{2}(\theta(r))}=1 \\
& \Leftrightarrow \\
& \left.\frac{m g f^{\prime \prime}(\theta) m g f(\theta)-\left(m g f^{\prime}(\theta)\right)^{2}}{m g f^{2}(\theta)}\right|_{\theta=\theta(r)}=\frac{1}{\theta^{\prime}(r)}
\end{aligned}
$$

Since $\left.\log (m g f(\theta))\right|_{\theta=\theta(r)}=r=E\left[\tilde{r}_{1}\right]$, we obtain $\sigma^{2}(r)=\left.\log "(m g f(\theta))\right|_{\theta=\theta(r)}=\frac{1}{\theta^{\prime}(r)}\left(=\frac{1}{\Lambda^{\prime \prime}(r)}\right)$.

Proof of Theorem 2. Given the probability space $\left(\Omega,\left\{\mathcal{F}_{n}\right\}_{n=1}^{\infty}\right)$ that generates the portfolio return $R_{p, n}^{(\boldsymbol{\alpha})}(\omega)$, where $(\omega)$ is used to emphasize that $R_{p, n}^{(\boldsymbol{\alpha})}(\omega)$ is a random variable, $(n)$ is used to stress that $R_{p, n}^{(\boldsymbol{\alpha})}(\omega)$ depends on $(n)$, and $(\boldsymbol{\alpha})$ is used to denote that $R_{p, n}^{(\boldsymbol{\alpha})}(\omega)$ depends on $(\boldsymbol{\alpha})$. The probability space satisfies the usual conditions as in Parthasarathy (1977, page 20). The proof of this theorem is divided in to two parts. 
- UPPER BOUND: Since

$$
\left\{\omega \in \Omega: R_{p, n}^{(\boldsymbol{\alpha})}(\omega) \geq r\right\} \equiv\left\{\omega \in \Omega: \exp \left\{n \theta R_{p, n}^{(\boldsymbol{\alpha})}(\omega)\right\} \geq \exp \{n \theta r\}\right\} \forall \theta>0
$$

then an application of the Tchebyshev inequality yields

$$
\begin{aligned}
P\left\{R_{p, n}^{(\boldsymbol{\alpha})}(\omega)>r\right\} & \leq P\left\{\exp \left\{n \theta R_{p, n}^{(\boldsymbol{\alpha})}(\omega)\right\}>\exp \{n \theta r\}\right\} \\
& \leq \exp \{-n \theta r\} E\left[\exp \left\{n \theta R_{p, n}^{(\boldsymbol{\alpha})}(\omega)\right\}\right]
\end{aligned}
$$

Hence, we have

$$
\limsup _{n \longrightarrow \infty} \frac{1}{n} \sup _{\boldsymbol{\alpha} \in S} \log P\left\{R_{p, n}^{(\boldsymbol{\alpha})}(\omega)>r\right\} \leq-\limsup _{n \longrightarrow \infty} \frac{1}{n} \inf _{\boldsymbol{\alpha} \in S}\left(n \hat{\theta} r-\log E\left[\exp \left\{n \hat{\theta} R_{p, n}^{(\boldsymbol{\alpha})}(\omega)\right\}\right]\right)
$$

where $S$ is a compact set of feasible investment and $\hat{\theta}$ is in the neighbourhood of

$$
\theta^{*}=\lim _{n \longrightarrow \infty} \frac{1}{n} \inf _{\boldsymbol{\alpha} \in S} \sup _{\theta \in(0, \infty)}\left(n \theta r-\log E\left[\exp \left\{n \theta R_{p, n}^{(\boldsymbol{\alpha})}(\omega)\right\}\right]\right)
$$

- LOWER BOUND: This part uses the Randon-Nykodym theorem and the Borell-Catelli lemma.

First, let us define another probability measure $Q$ on the probability space $\left(\Omega,\left\{\mathcal{F}_{n}\right\}_{n=1}^{\infty}\right)$ such that

$$
\left.\frac{d Q}{d P}\right|_{\mathcal{F}_{n}} \stackrel{\text { def }}{=} \exp \left\{n \hat{\theta} R_{p, n}^{(\hat{\boldsymbol{\alpha}})}(\omega)-\log E\left[\exp \left\{n \hat{\theta} R_{p, n}^{(\hat{\boldsymbol{\alpha}})}(\omega)\right\}\right]\right\}
$$

where

$$
\hat{\boldsymbol{\alpha}}(\hat{\theta}, n)=\operatorname{arginf}_{\boldsymbol{\alpha} \in S}\left(n \hat{\theta} r-\log E\left[\exp \left\{n \hat{\theta} R_{p, n}^{(\boldsymbol{\alpha})}(\omega)\right\}\right]\right)
$$

Next, since $\left\{\omega \in \Omega: R_{p, n}^{(\hat{\boldsymbol{\alpha}})}(\omega)>r-\epsilon\right\} \supseteq\left\{\omega \in \Omega: r-\epsilon<R_{p, n}^{(\hat{\boldsymbol{\alpha}})}(\omega)<r+\epsilon\right\}$, we can 
deduce

$$
\begin{aligned}
& \log P\left\{R_{n, p}^{(\hat{\boldsymbol{\alpha}})}(\omega)>r-\epsilon\right\} \\
\geq & \log P\left\{r-\epsilon<R_{p, n}^{(\hat{\boldsymbol{\alpha}})}(\omega)<r+\epsilon\right\} \\
= & \log \int_{\omega \in \Omega} \frac{d P}{d Q} I_{\left\{\omega \in \Omega: r-\epsilon<R_{p, n}^{(\hat{\boldsymbol{\alpha}})}(\omega)<r+\epsilon\right\}}(\omega) d Q \\
= & \log \int_{\omega \in \Omega} \exp \left\{\log E\left[\exp \left\{n \hat{\theta} R_{p, n}^{(\hat{\boldsymbol{\alpha}})}(\omega)\right\}\right]-n \hat{\theta} R_{p, n}^{(\hat{\boldsymbol{\alpha}})}(\omega)\right\} I_{\left\{\omega \in \Omega: r-\epsilon<R_{p, n}^{(\hat{\boldsymbol{\alpha}})}(\omega)<r+\epsilon\right\}}(\omega) d Q \\
\geq & \log \int_{\omega \in \Omega} \exp \left\{\log E\left[\exp \left\{n \hat{\theta} R_{p, n}^{(\hat{\boldsymbol{\alpha}})}(\omega)\right\}\right]-n \hat{\theta}(r+\epsilon)\right\} I_{\left\{\omega \in \Omega: r-\epsilon<R_{p, n}^{(\hat{\boldsymbol{\alpha}})}(\omega)<r+\epsilon\right\}}(\omega) d Q \\
= & -n \hat{\theta}(r+\epsilon)+\log E\left[\exp \left\{n \hat{\theta} R_{p, n}^{(\hat{\boldsymbol{\alpha}})}(\omega)\right\}\right]+\log \int_{\omega \in \Omega} I_{\left\{\omega \in \Omega: r-\epsilon<R_{p, n}^{(\hat{\boldsymbol{\alpha}})(\omega)<r+\epsilon\}}\right.}(\omega) d Q \\
= & -n \hat{\theta} r+\log E\left[\exp \left\{n \hat{\theta} R_{p, n}^{(\hat{\boldsymbol{\alpha}})}(\omega)\right\}\right]+\log Q\left\{r-\epsilon<R_{p, n}^{(\hat{\boldsymbol{\alpha}})}(\omega)<r+\epsilon\right\}-n \hat{\theta} \epsilon .
\end{aligned}
$$

Hence,

$$
\begin{aligned}
\liminf _{n \longrightarrow \infty} \frac{1}{n} \sup _{\boldsymbol{\alpha} \in S} \log P\left\{R_{p, n}^{(\hat{\boldsymbol{\alpha}})}(\omega)>r-\epsilon\right\} & \geq-\liminf _{n \longrightarrow \infty} \frac{1}{n}\left(n \hat{\theta} r-\log E\left[\exp \left\{n \hat{\theta} R_{p, n}^{(\hat{\boldsymbol{\alpha}})}(\omega)\right\}\right]\right) \\
& +\liminf _{n \longrightarrow \infty} \frac{1}{n} \log Q\left\{r-\epsilon<R_{p, n}^{(\hat{\boldsymbol{\alpha}})}(\omega)<r+\epsilon\right\}-o(1) .
\end{aligned}
$$

Thus, the lower bound is obtained if we can show that

$$
\liminf _{n \longrightarrow \infty} \frac{1}{n} \log Q\left\{r-\epsilon<R_{p, n}^{(\hat{\alpha})}(\omega)<c+\epsilon\right\}=0 .
$$

In order to show $(\mathrm{A.43})$, we need to define the event

$$
A_{n}(\omega) \stackrel{\text { def }}{=}\left\{\omega \in \Omega: r-\epsilon<R_{p, n}^{(\hat{\boldsymbol{\alpha}})}(\omega)<r+\epsilon\right\}=\left\{\omega \in \Omega:\left|R_{p, n}^{(\hat{\boldsymbol{\alpha}})}(\omega)-r\right|<\epsilon\right\} .
$$

Since $R_{p, n}^{(\hat{\boldsymbol{\alpha}})}(\omega)$ has the asymmetric Gamma distribution, thus $P\left\{A_{n}(\omega)\right\} \longrightarrow 0$ as $\epsilon$ is arbitrarily small, and $(n)$ is sufficiently large. (See Figure iii for an illustration.) On the other hand, since $\exp \left\{n \hat{\theta} R_{p, n}^{(\hat{\boldsymbol{\alpha}})}(\omega)-\log E\left[\exp \left\{n \hat{\theta} R_{p, n}^{(\hat{\boldsymbol{\alpha}})}(\omega)\right\}\right]\right\}$ is always positive and infinitely differentiable, the conjugate measure $(Q)$ is absolutely continuous w.r.t. $(P)$. 
[See Parthasarathy (1977) for definition of absolute continuity.] Then, we have

$$
\lim _{n \longrightarrow \infty} Q\left\{A_{n}(\omega)\right\} \longrightarrow 0
$$

Thus, $\sum_{n=1}^{\infty} Q\left\{A_{n}(\omega)\right\} \leq \infty$, whereby an application of the Borel-Catelli lemma yields

$$
Q\left\{\bigcap_{m=1}^{\infty} \bigcup_{n=m}^{\infty} A_{n}(\omega)\right\}=Q\left\{\limsup _{n \longrightarrow \infty} A_{n}(\omega)\right\}=0
$$

which is also equivalent to

$$
Q\left\{\bigcup_{m=1}^{\infty} \bigcap_{n=m}^{\infty} A_{n}(\omega)\right\}=Q\left\{\liminf _{n \longrightarrow \infty} A_{n}(\omega)\right\}=1
$$

Moreover, since $\left\{\omega \in \Omega: r-\epsilon<R_{p, n}^{(\hat{\boldsymbol{\alpha}})}(\omega)<r+\epsilon\right\} \supseteq \inf _{n \in(0, \infty)}\left\{\omega \in \Omega: r-\epsilon<R_{p, n}^{(\hat{\boldsymbol{\alpha}})}(\omega)<\right.$ $r+\epsilon\}$, we obtain

$$
\frac{1}{n} \log Q\left\{r-\epsilon<R_{p, n}^{(\hat{\boldsymbol{\alpha}})}(\omega)<r+\epsilon\right\} \geq \frac{1}{n} \log Q\left\{\liminf _{n \longrightarrow \infty}\left\{\omega \in \Omega: r-\epsilon<R_{p, n}^{(\hat{\boldsymbol{\alpha}})}(\omega)<r+\epsilon\right\}\right\}
$$

which is equivalent to

$$
\begin{aligned}
\liminf _{n \longrightarrow \infty} \frac{1}{n} \log Q\left\{r-\epsilon<R_{p, n}^{(\hat{\boldsymbol{\alpha}})}(\omega)<r+\epsilon\right\} & =\lim _{n \longrightarrow \infty} \frac{1}{n} \log Q\left\{\liminf _{n \longrightarrow \infty} A_{n}(\omega)\right\} \\
& \longrightarrow 0
\end{aligned}
$$

To complete the proof, we still need to show that

$$
\limsup _{n \longrightarrow \infty} \frac{1}{n}\left(n \hat{\theta} r-\log E\left[\exp \left\{n \hat{\theta} R_{p, n}^{(\hat{\boldsymbol{\alpha}})}(\omega)\right\}\right]\right)=\liminf _{n \longrightarrow \infty} \frac{1}{n}\left(n \hat{\theta} r-\log E\left[\exp \left\{n \hat{\theta} R_{p, n}^{(\hat{\boldsymbol{\alpha}})}(\omega)\right\}\right]\right) .
$$

First, since

$$
\Lambda_{n}(\hat{\theta}, r)=\frac{1}{n} \log \left\{\frac{\exp \{n \hat{\theta} r\}}{E\left[\exp \left\{n \hat{\theta} R_{p, n}^{(\hat{\boldsymbol{\alpha}})}(\omega)\right\}\right]}\right\}=\log \left\{E\left[\exp \left\{n \hat{\theta}\left(R_{p, n}^{(\hat{\boldsymbol{\alpha}})}(\omega)-r\right)\right\}\right]\right\}^{-\frac{1}{n}}
$$


where $\log \left\{E\left[\exp \left\{n \hat{\theta}\left(R_{p, n}^{(\hat{\boldsymbol{\alpha}})}(\omega)-r\right)\right\}\right]\right\}^{-\frac{1}{n}}$ is a convex function. An application of Jensen's inequality yields

$$
\Lambda_{n}(\hat{\theta}, r) \leq \log E\left[\exp \left\{-\hat{\theta}\left(R_{p, n}^{(\hat{\boldsymbol{\alpha}})}(\omega)-r\right)\right\}\right]
$$

Since the function $\exp \left\{-\hat{\theta}\left(R_{p, n}^{(\hat{\boldsymbol{\alpha}})}(\omega)-r\right)\right\}$ is $\mathcal{F}_{n}$ measurable, an application of the Fatou lemma yields

$$
\begin{aligned}
\limsup _{n \longrightarrow \infty} \Lambda_{n}(\hat{\theta}, r) & \leq \log \limsup _{n \longrightarrow \infty} E\left[\exp \left\{-\hat{\theta}\left(R_{p, n}^{(\hat{\boldsymbol{\alpha}})}(\omega)-r\right)\right\}\right] \\
& \leq \log E\left[\limsup _{n \longrightarrow \infty} \exp \left\{-\hat{\theta}\left(R_{p, n}^{(\hat{\boldsymbol{\alpha}})}(\omega)-r\right)\right\}\right]
\end{aligned}
$$

Second, since $\left\{\omega \in \Omega: R_{p, n-1}^{(\hat{\boldsymbol{\alpha}})}(\omega)=R\right\} \subset\left\{\omega \in \Omega: R_{p, n}^{(\hat{\boldsymbol{\alpha}})}(\omega)=R\right\}$ or $\mathcal{F}_{n-1} \subset \mathcal{F}_{n}$ and $f: R \longrightarrow \exp \{\hat{\theta} R\}$ is one-to-one mapping, the random sequence $\left\{\omega \in \Omega: \exp \left\{-\hat{\theta}\left(R_{p, i}^{(\hat{\boldsymbol{\alpha}})}(\omega)-\right.\right.\right.$ $r)\}=R\}_{i=0}^{n}$ is monotonically increasing. Then, we have

$$
\begin{aligned}
\left\{\omega \in \Omega: \limsup _{n \longrightarrow \infty} \exp \left\{-\hat{\theta}\left(R_{p, n}^{(\hat{\boldsymbol{\alpha}})}(\omega)-r\right)\right\}\right\} & =\left\{\omega \in \Omega: \liminf _{n \longrightarrow \infty} \exp \left\{-\hat{\theta}\left(R_{p, n}^{(\hat{\boldsymbol{\alpha}})}(\omega)-r\right)\right\}\right\} \\
& =\left\{\omega \in \Omega: \lim _{n \longrightarrow \infty} \exp \left\{-\hat{\theta}\left(R_{p, n}^{(\hat{\boldsymbol{\alpha}})}(\omega)-r\right)\right\}\right\}
\end{aligned}
$$

Another application of the Fatou lemma yields

$$
\limsup _{n \longrightarrow \infty} \Lambda_{n}(\hat{\theta}, r) \leq \liminf _{n \longrightarrow \infty} \Lambda_{n}(\hat{\theta}, r)
$$

On the other hand, it is obvious that

$$
\limsup _{n \longrightarrow \infty} \Lambda_{n}(\hat{\theta}, r) \geq \liminf _{n \longrightarrow \infty} \Lambda_{n}(\hat{\theta}, r)
$$

Thus, (A.44) is shown. In view of (A.43) and (A.44), we have proved Theorem 2. 


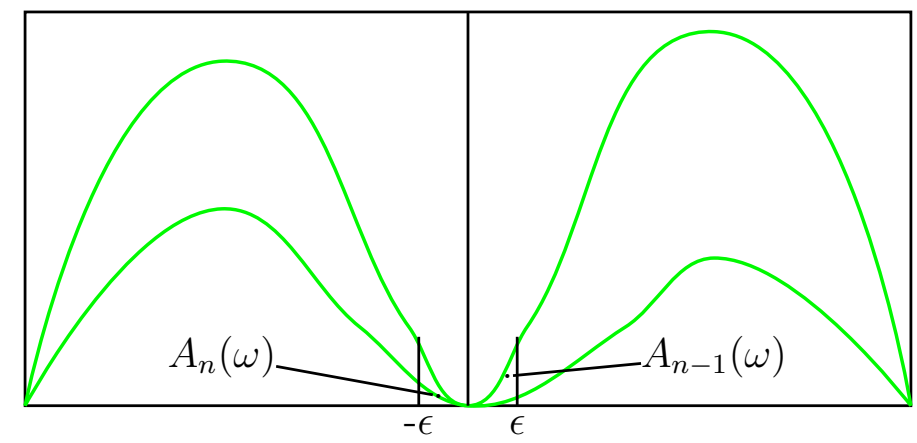

Figure iii: The asymmetric Gamma distribution $\left(A_{n}(\omega)\right.$ shrinks as $n \longrightarrow \infty$ and $\left.\epsilon \longrightarrow 0\right)$

\section{Appendix B. Nonlinear Correlations}

The third order Edgeworth expansion of the deviations function is given by

$$
\begin{aligned}
\Lambda(r, \theta, \alpha) & =r \theta-\left\{\theta\left(\alpha \mu_{1}+(1-\alpha) \mu_{2}\right)+\frac{\theta^{2}}{2}\left(\alpha^{2} \sigma_{1}^{2}+(1-\alpha)^{2} \sigma_{2}^{2}+2 \alpha(1-\alpha) \lambda_{11}\right)\right. \\
& \left.+\frac{\theta^{3}}{6}\left(\alpha^{3} \lambda_{1}+(1-\alpha)^{3} \lambda_{2}+3 \alpha^{2}(1-\alpha) \lambda_{12}+3 \alpha(1-\alpha)^{2} \lambda_{21}\right)\right\},
\end{aligned}
$$

where $\mu_{i}, \sigma_{i}$, and $\lambda_{i}$ for $i=1,2$ are the means, the variances, and the third central moments resp. $\lambda_{11}, \lambda_{12}$, and $\lambda_{21}$ are the linear correlation and the nonlinear correlations resp. The first nonlinear correlation is the error coefficient obtained when the first asset return is regressed on the squared second asset return. That is, supposing that the means of the asset returns are zero, i.e. $E\left[\tilde{r}_{1}\right]=E\left[\tilde{r}_{2}\right]=0$, then $\tilde{r}_{1}$ is regressed on $\tilde{r}_{2}^{2}$. This regression gives the mean square error(MSE) as follows:

$$
\begin{aligned}
M S E & =\frac{1}{T} \sum_{t=1}^{T}\left(\tilde{r}_{1 t}-b \tilde{r}_{2 t}^{2}\right)^{2} \\
& =\frac{1}{T} \sum_{t=1}^{T} \tilde{r}_{2}^{4}\left(b-\frac{\frac{1}{T} \sum_{t=1}^{T} \tilde{r}_{1 t} \tilde{r}_{2 t}^{2}}{\frac{1}{T} \sum_{t=1}^{T} \tilde{r}_{2 t}^{4}}\right)^{2} \\
& +\frac{1}{T} \sum_{t=1}^{T} \tilde{r}_{1 t}\left[1-\left(\frac{\frac{1}{T} \sum_{t=1}^{T}\left(\tilde{r}_{1 t} \tilde{r}_{2 t}^{2}\right)}{\sqrt{\left(\frac{1}{T} \sum_{t=1}^{T} \tilde{r}_{1 t}^{2}\right)\left(\frac{1}{T} \sum_{t=1}^{T} \tilde{r}_{2 t}^{4}\right)}}\right)^{2}\right] .
\end{aligned}
$$

Hence, the LS estimate of $(b)$ is

$$
\widehat{b}_{T}=\frac{\sum_{t=1}^{T}\left(\tilde{r}_{1 t} \tilde{r}_{2 t}^{2}\right)}{\sum_{t=1}^{T} \tilde{r}_{2 t}^{4}},
$$


and the minimum MSE is given by

$$
\frac{1}{T} \sum_{t=1}^{T} \tilde{r}_{1 t}\left[1-\left(\frac{\frac{1}{T} \sum_{t=1}^{T}\left(\tilde{r}_{1 t} \tilde{r}_{2 t}^{2}\right)}{\sqrt{\left(\frac{1}{T} \sum_{t=1}^{T} \tilde{r}_{1 t}^{2}\right)\left(\frac{1}{T} \sum_{t=1}^{T} \tilde{r}_{2 t}^{4}\right)}}\right)^{2}\right],
$$

whereby we define $\widehat{\lambda}_{12}=\frac{\sum_{t=1}^{T}\left(\tilde{r}_{1 t} \tilde{r}_{2 t}^{2}\right)}{\sqrt{\sum_{t=1}^{T} \tilde{r}_{1 t}^{2} \sum_{t=1}^{T} \tilde{r}_{2 t}^{4}}}$ as the sample estimate of the first measure of nonlinear correlation. If $\widehat{\lambda}_{12}$ is equal to -1 or 1 , the actual data are well fitted in to the horizontal parabolic curve or the vertical one. (See Figure iv.)

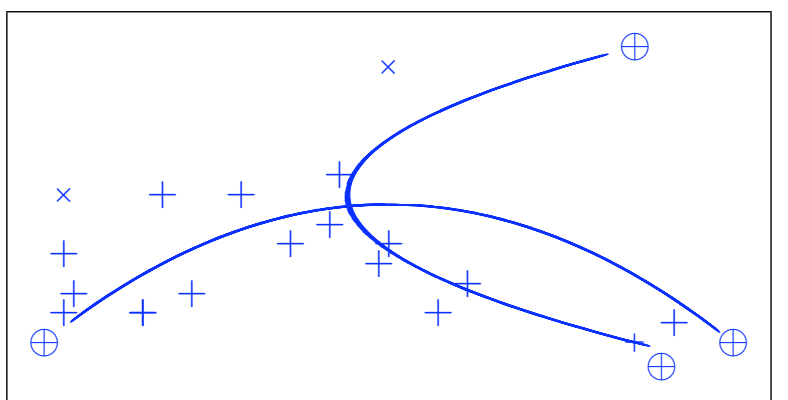

Figure iv: Measures of nonlinear correlation measure asymmetric dependence

It follows that $\left|\widehat{\lambda}_{12}\right|$ must be less than 1. Furthermore, the nonlinear correlations illustrate the strength of the nonlinear relationships between $\tilde{r}_{1}$ and $\tilde{r}_{2}$ and vice versa. Since the nonlinear relationship between $\tilde{r}_{1}$ and $\tilde{r}_{2}$ (as gauged by the first nonlinear correlation) is different from the nonlinear relationship between $\tilde{r}_{2}$ and $\tilde{r}_{1}$ (as gauged by the second nonlinear correlation). The nonlinear correlations measure asymmetric dependence. Even when the conventional linear correlation between $\tilde{r}_{1}$ and $\tilde{r}_{2}$ is zero, there can still be nonzero nonlinear correlations between $\tilde{r}_{1}$ and $\tilde{r}_{2}$. 


\section{References}

Bawa, V. S. (1978). Safety-first, stochastic dominance, and optimal portfolio choice, Journal of Financial and Quantitative Analysis 13: 255-271.

Burden, R. and Fairs, J. (1997). Numerical Analysis, Brooks/Cole Publishing Company, California.

Cramer, H. (1946). Mathematical Methods of Statistics, Princeton University Press, Princeton.

Dembo, A. and Zeitouni, O. (1999). Large Deviations Techniques and Applications, Jones and Bartlett Publishers, Boston, London.

Fan, J. and Gu, J. (2003). Semiparametric estimation of Value at Risk, Econometrics Journal 6: $261-290$.

Harlow, W. and Rao, K. S. (1989). Asset pricing in a generalized mean-lower partial moment framework: theory and evidence, Journal of Financial and Quantitative Analysis 24(3): 285311.

Harvey, C. R. and Siddique, A. (2000). Conditional skewness in asset pricing tests, Journal of Finance 3: 1263-1295.

Ingersoll, J. E. (1987). Theory of Financial Decision Making, Rowman \& Littlefield, New York.

Jondeau, E. and Rockinger, M. (1999). Estimating Gram-Charlier expansions with positivity constraints, working paper, Banque de France.

Jondeau, E. and Rockinger, M. (2003). Conditional volatility, skewness, and kurtosis: Existence, persistence, and comovement, Journal of Economic Dynamics and Control 27: 1699-1737.

Kane, A. (1982). Skewness preference and portfolio choice, Journal of Financial and Quatitative Analysis 17(1): 15-25. 
Knight, J. L., Satchell, S. and Tran, K. (1995). Statistical modelling of asymmetric risk in asset returns, Applied Mathematical Finance 3: 155-172.

Malevergne, Y. and Sornette, D. (2002). Multi-moments methods for portfolio management: Generalized CAPM in homogeneous and heterogeneous markets, working paper .

Markowitz, H. (1952). Portfolio selection, Journal of Finance 7(1): 77-91.

Menezes, C., Geiss, C. and Tressler, J. (1980). Increasing downside risk, American Economic Review 70(5): 921-932.

Parthasarathy, K. R. (1977). Introduction to Probability and Measure, The MacMillan Company of India Ltd, Calcutta.

Patton, A. J. (2004). On the importance of skewness and asymmetric dependence in stock returns for asset allocation, Journal of Financial Econometrics 2: 130-168.

Rao, C. R. (1973). Linear Statistical Inference and Its Applications, John Wiley \& Sons, New York.

Scales, L. E. (1985). Introduction to Nonlinear Optimization, MacMillan Publishers Ltd, London.

Shiryaev, A. N. (1995). Probability, Vol. 95 of Graduate text in mathematics, 2 edn, SpringerVerlag, New York Berlin Heidelberg.

Stutzer, M. (2000). A portfolio performance index, Financial Analysts Journal 56.

Stutzer, M. (2001a). A large deviation approach to portfolio analysis, working paper, University of Iowa .

Stutzer, M. (2001b). Optimal asset allocation for endowments: A large deviation approach, working paper, University of Iowa.

Stutzer, M. (2003). Portfolio choice with endogeneous utility: A large deviation approach, Journal of Econometrics 116: 365-386. 
Stutzer, M. (2004). Asset allocation without unobservable parameters, Financial Analysts Journal 60(5): 38-51.

Wong, C. M. and So, M. K. P. (2004). On conditional moments of GARCH models, with applications to multiple period value, Statistica Sinica 13: 1015-1044.

Zhulenev, S. V. (1997). On the large deviation, Theory of Probability and Its Applications 44(1): $75-92$. 
Table 3: Sample statistics

\begin{tabular}{lcc}
\hline Stock & Bangkok SET & S\&P 500 \\
\hline Minimal value & -0.2997 & -0.2153 \\
Maximal value & 0.3287 & 0.1346 \\
5\% Quantile & -0.1311 & -0.0558 \\
95\% Quantile & 0.1502 & 0.0760 \\
Mean & 0.0074 & 0.0126 \\
Standard deviation & 0.0088 & 0.0429 \\
Skewness & 0.3232 & -0.5249 \\
Kurtosis & 5.3214 & 5.6127 \\
Linear correlation & 0.2625 & 0.2625 \\
First sample nonlinear correlation $\left(\widehat{\lambda}_{12}\right)$ & 0.3637 & \\
Second sample nonlinear correlation $\left(\widehat{\lambda}_{21}\right)$ & & 0.2771 \\
\hline
\end{tabular}


Table 4: The ML estimates of the parameters of the return distributions (the parenthetical numbers are standard errors)

\begin{tabular}{cccccc}
\hline \multirow{2}{*}{ Stock } & \multicolumn{2}{c}{ Asymmetric Gamma distribution } & & \multicolumn{2}{c}{ Partially) symmetric Gamma distribution } \\
\cline { 2 - 3 } \cline { 5 - 6 } & Bangkok SET & S\&P 500 & & Bangkok SET & S\&P 500 \\
\hline$\kappa_{1}$ & $0.9577(0.1105)$ & $1.5945(0.0488)$ & & $1.0152(1.4711)$ & $1.5799(1.9216)$ \\
$\kappa_{2}$ & $0.9902(0.3629)$ & $1.1912(2.1240)$ & & $0.9243(1.3685)$ & $1.2085(1.8402)$ \\
$\gamma_{1}$ & $0.0698(1.6560)$ & $0.0236(2.6076)$ & & $0.0634(0.1015)$ & $0.0239(0.0337)$ \\
$\gamma_{2}$ & $0.0565(0.1565)$ & $0.0244(0.0453)$ & & & \\
Shifting probability & 0.5146 & 0.6254 & & 0.5146 & 0.6254 \\
Mean & 0.0072 & 0.1266 & & 0.0046 & 0.0128 \\
Standard deviation & 0.0039 & 0.0008 & & 0.0062 & 0.0287 \\
Skewness & 0.6581 & 0.5603 & & 0.1544 & -0.6191 \\
Kurtosis & 6.2156 & 5.0804 & & 6.4179 & 6.6318 \\
\hline
\end{tabular}


Table 5: The LR test for asymmetry in the return distributions Stock LR value Result

BangKok SET $33 \times 10^{-43}$ Accept symmetry

S\&P $500 \quad 12 \times 10^{-34}$ Accept symmetry


Table 6: Optimal portfolios and the risk bounds for different models

\begin{tabular}{|c|c|c|c|c|c|c|c|c|c|c|c|c|c|c|}
\hline \multirow{3}{*}{ Model } & \multicolumn{12}{|c|}{$\hat{\theta}$ (the Arrow-Pratt measure of absolute risk aversion) } & \multirow{2}{*}{\multicolumn{2}{|c|}{ 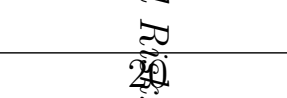 }} \\
\hline & \multicolumn{2}{|c|}{8} & \multicolumn{2}{|c|}{10} & \multicolumn{2}{|c|}{12} & \multicolumn{2}{|c|}{14} & \multicolumn{2}{|c|}{16} & \multicolumn{2}{|c|}{18} & & \\
\hline & $\bar{\alpha}(\hat{\theta})^{+}$ & Risk $^{++}$ & $\hat{\alpha}(\hat{\theta})$ & Risk & $\hat{\alpha}(\hat{\theta})$ & Risk & $\hat{\alpha}(\hat{\theta})$ & Risk & $\hat{\alpha}(\hat{\theta})$ & Risk & $\hat{\alpha}(\hat{\theta})$ & Risk & $\hat{\alpha}(\hat{\theta})$ & Risk \\
\hline $\mathbb{M}_{1}$ & 0.9678 & 0.4168 & 0.9396 & 0.3374 & 0.9208 & 0.2739 & 0.9070 & 0.2230 & 0.8973 & 0.1820 & 0.8890 & 0.1490 & 0.8832 & 0.1223 \\
\hline $\mathbb{M}_{2}$ & 0.9896 & 0.4159 & 0.9652 & 0.3361 & 0.9500 & 0.2722 & 0.9402 & 0.2209 & 0.9338 & 0.1795 & 0.9298 & 0.1446 & 0.9276 & 0.1191 \\
\hline $\mathbb{M}_{3}$ & 0.8678 & 0.1618 & 0.8695 & 0.1338 & 0.8487 & 0.0911 & 0.8396 & 0.0627 & 0.8295 & 0.0436 & 0.8195 & 0.0305 & 0.7988 & 0.0216 \\
\hline $\mathbb{M}_{4}$ & 0.5740 & 0.1925 & 0.5521 & 0.1306 & 0.5641 & 0.0880 & 0.5117 & 0.0612 & 0.5519 & 0.0412 & 0.5719 & 0.0283 & 0.6091 & 0.0195 \\
\hline $\mathbb{M}_{5}$ & 0.6763 & 0.1911 & 0.6768 & 0.1275 & 0.6068 & 0.0863 & 0.5991 & 0.0582 & 0.5994 & 0.0394 & 0.6000 & 0.0267 & 0.6894 & 0.0181 \\
\hline $\mathbb{M}_{6}$ & 0.6100 & 0.1990 & 0.6091 & 0.1374 & 0.5999 & 0.0955 & 0.6071 & 0.0671 & 0.5938 & 0.0480 & 0.6067 & 0.0352 & 0.6414 & 0.0256 \\
\hline $\mathbb{M}_{7}$ & 0.5180 & 0.1975 & 0.4731 & 0.1349 & 0.5103 & 0.0911 & 0.6428 & 0.0596 & 0.8606 & 0.0370 & 0.7152 & 0.0271 & 0.5181 & 0.0379 \\
\hline $\mathbb{M}_{8}$ & 0.5211 & 0.1961 & 0.5210 & 0.1322 & 0.5282 & 0.0884 & 0.5205 & 0.0609 & 0.5128 & 0.0423 & 0.7166 & 0.0259 & 0.5272 & 0.0379 \\
\hline $\mathbb{M}_{9}$ & 0.5979 & 0.2011 & 0.5982 & 0.1382 & 0.5983 & 0.0963 & 0.5984 & 0.0681 & 0.5982 & 0.0491 & 0.5985 & 0.0362 & 0.5985 & 0.0276 \\
\hline
\end{tabular}

$+\hat{\alpha}(\hat{\theta})$ is the optimal portfolio for a given $\hat{\theta}$.

${ }^{++}$Risk is defined as the upper bound of $P\left\{R_{p, 2}^{(\alpha)}(\omega)<-1 \%\right\}$ for a given $\hat{\theta}$. 
Table 7: The means, the standard deviations, and the skewnesses of the optimal portfolio returns obtained in the different models for $\hat{\theta}_{A P}=20$

\begin{tabular}{cccccccccc}
\hline Model & $\mathbb{M}_{1}$ & $\mathbb{M}_{2}$ & $\mathbb{M}_{3}$ & $\mathbb{M}_{4}$ & $\mathbb{M}_{5}$ & $\mathbb{M}_{6}$ & $\mathbb{M}_{7}$ & $\mathbb{M}_{8}$ & $\mathbb{M}_{9}$ \\
\hline$\hat{\alpha}$ & 0.8832 & 0.9276 & 0.7988 & 0.6091 & 0.6894 & 0.6414 & 0.5181 & 0.5272 & 0.5985 \\
Portfolio mean & 0.0120 & 0.0122 & 0.0115 & 0.0106 & 0.0110 & 0.0108 & 0.0084 & 0.0089 & 0.0102 \\
Portfolio standard deviation & 0.0264 & 0.0272 & 0.0262 & 0.0344 & 0.0319 & 0.0334 & 0.0379 & 0.0374 & 0.0348 \\
Portfolio skewness & 0.5109 & 0.5399 & 0.4506 & 0.6038 & 0.5415 & 0.5636 & 0.4519 & 0.4761 & 0.5645 \\
\hline
\end{tabular}


Figure 1: Plots of the kernel densities of asset returns

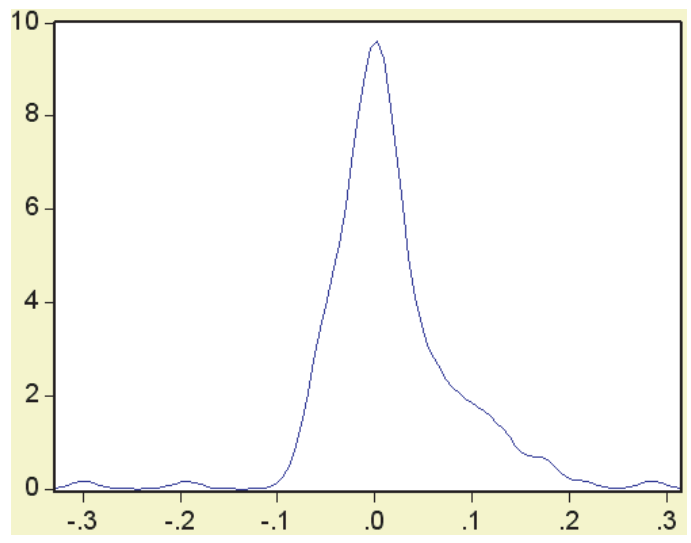

(a) Bangkok SET

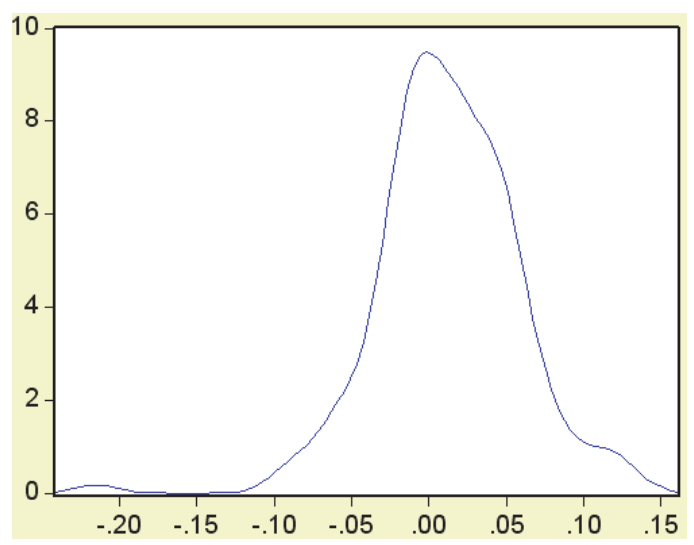

(b) $\mathrm{S} \& \mathrm{P} 500$

Figure 2: Plots of Equation 10

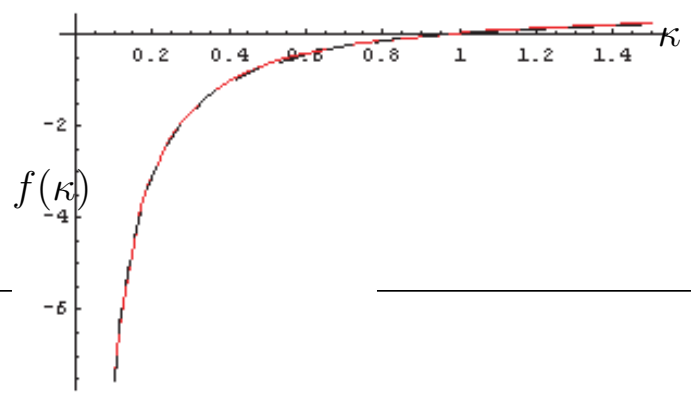

(a) Bangkok SET

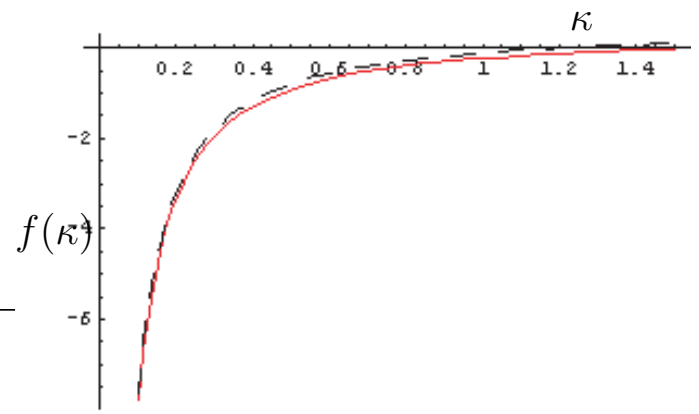

(b) $\mathrm{S} \& \mathrm{P} 500$

Figure 3: The simulated relationship between the optimal portfolio and the recovered linear correlation $\left(\hat{\lambda}_{11}\right)$
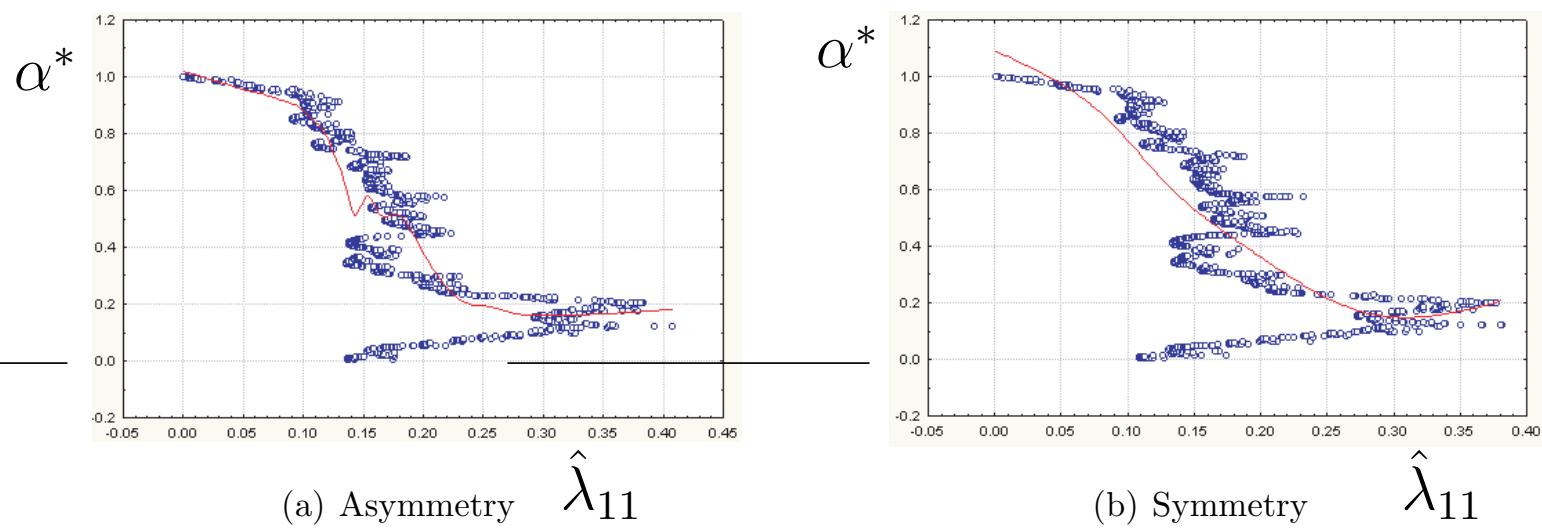
Figure 4: The simulated relationship between the optimal portfolio and the recovered nonlinear correlations $\left(\hat{\lambda}_{12}\right.$ and $\left.\hat{\lambda}_{21}\right)$

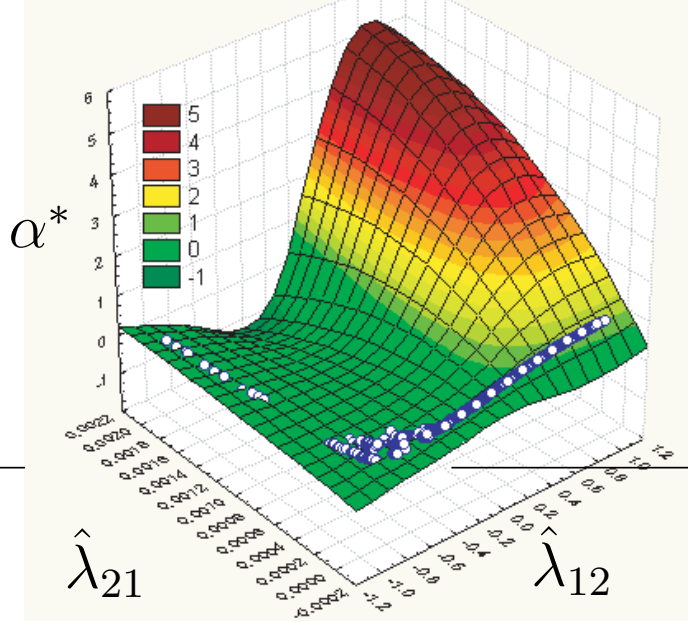

(a) Asymmetry

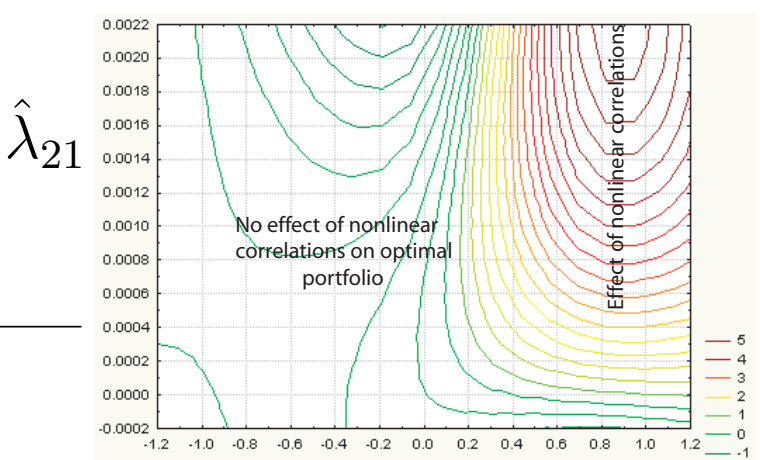

$\hat{\lambda}_{12}$

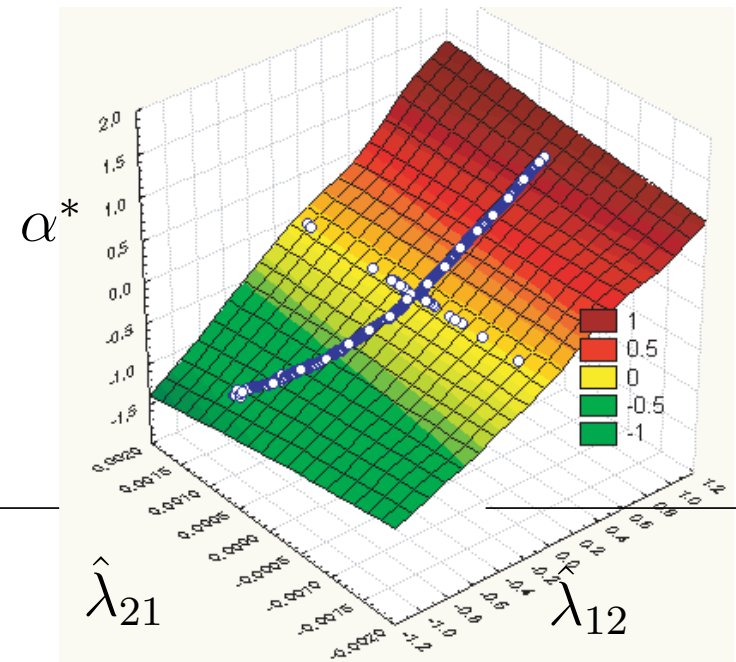

(b) Symmetry

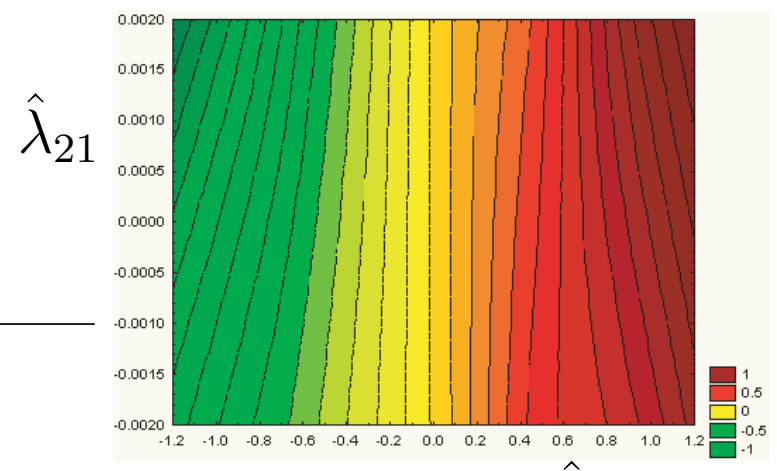

$\hat{\lambda}_{12}$ 
Figure 5: The deviations functions derived from the symmetric return distributions when two assets are uncorrelated (the benchmark return $r=1 \%$ )

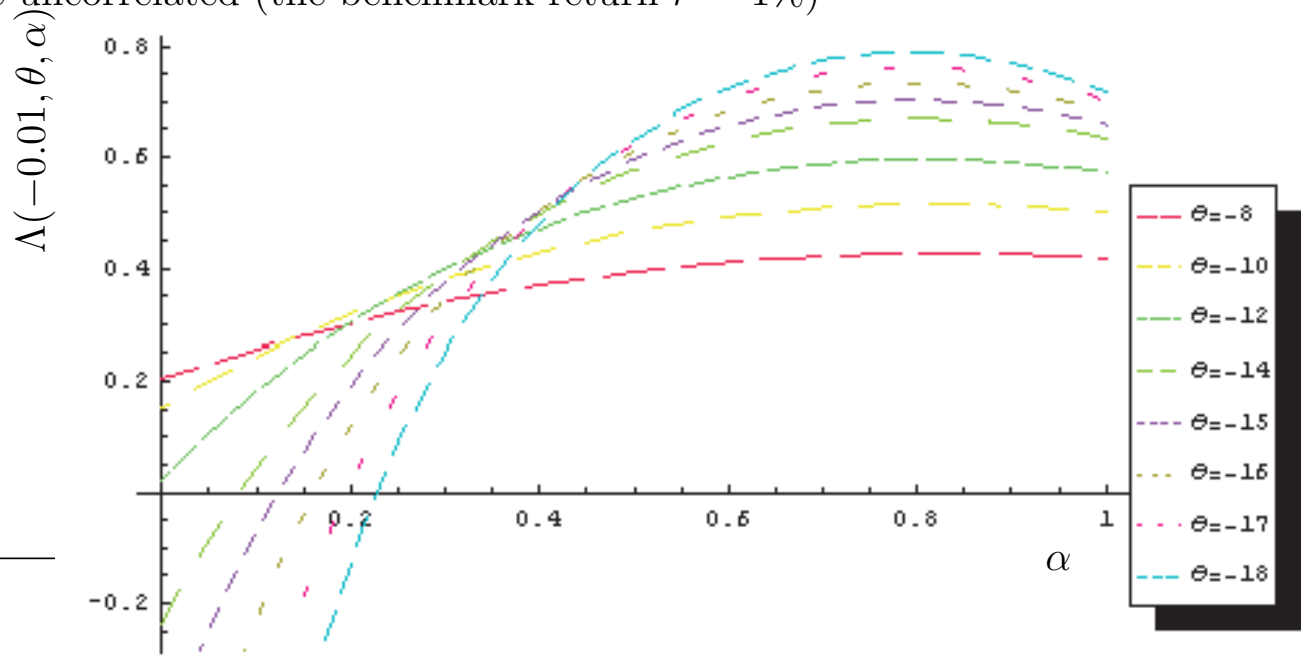

Figure 6: The deviations functions derived from the asymmetric portfolio return distribution (the benchmark return $r=-1 \%$ )

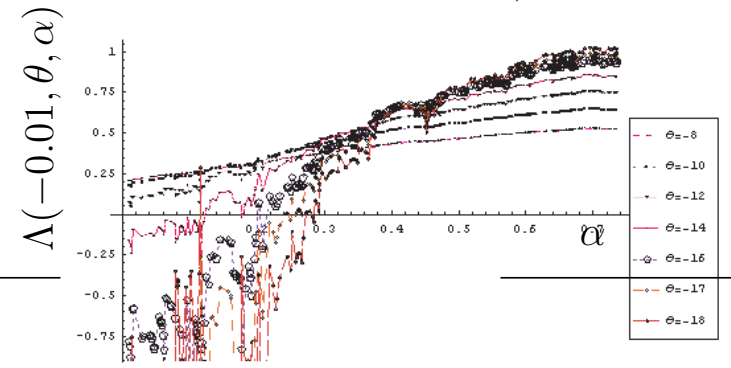

(a) The deviations functions obtained by simulations

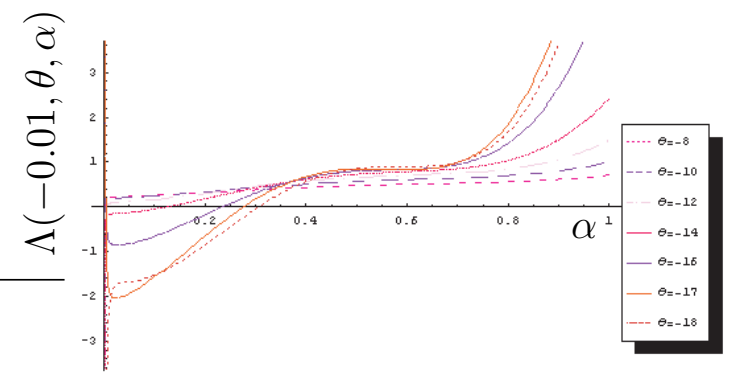

(b) The deviations functions obtained by a cubic spline interpolation

Figure 7: The deviations functions derived from the partially symmetric portfolio return distribution (the benchmark return $r=-1 \%$ )

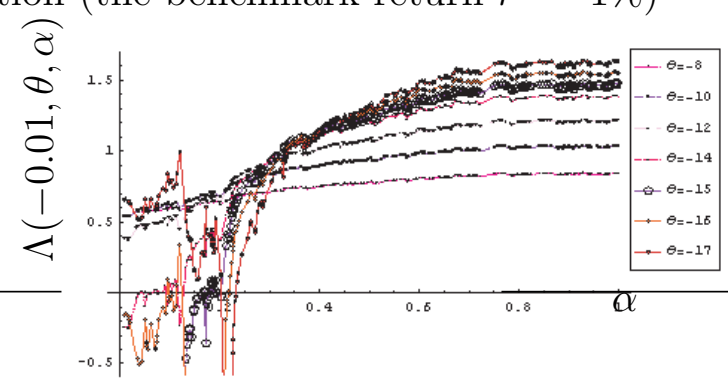

(a) The deviations functions obtained by simulations

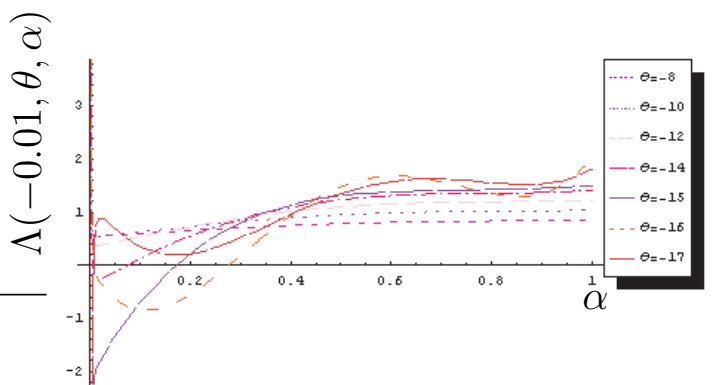

(b) The deviations functions obtained by a cubic spline interpolation 


\section{List of other working papers:}

\section{6}

1. Roman Kozhan, Multiple Priors and No-Transaction Region, WP06-24

2. Martin Ellison, Lucio Sarno and Jouko Vilmunen, Caution and Activism? Monetary Policy Strategies in an Open Economy, WP06-23

3. Matteo Marsili and Giacomo Raffaelli, Risk bubbles and market instability, WP06-22

4. Mark Salmon and Christoph Schleicher, Pricing Multivariate Currency Options with Copulas, WP06-21

5. Thomas Lux and Taisei Kaizoji, Forecasting Volatility and Volume in the Tokyo Stock Market: Long Memory, Fractality and Regime Switching, WP06-20

6. Thomas Lux, The Markov-Switching Multifractal Model of Asset Returns: GMM Estimation and Linear Forecasting of Volatility, WP06-19

7. Peter Heemeijer, Cars Hommes, Joep Sonnemans and Jan Tuinstra, Price Stability and Volatility in Markets with Positive and Negative Expectations Feedback: An Experimental Investigation, WP06-18

8. Giacomo Raffaelli and Matteo Marsili, Dynamic instability in a phenomenological model of correlated assets, WP06-17

9. Ginestra Bianconi and Matteo Marsili, Effects of degree correlations on the loop structure of scale free networks, WP06-16

10. Pietro Dindo and Jan Tuinstra, A Behavioral Model for Participation Games with Negative Feedback, WP06-15

11. Ceek Diks and Florian Wagener, A weak bifucation theory for discrete time stochastic dynamical systems, WP06-14

12. Markus Demary, Transaction Taxes, Traders' Behavior and Exchange Rate Risks, WP06-13

13. Andrea De Martino and Matteo Marsili, Statistical mechanics of socio-economic systems with heterogeneous agents, WP06-12

14. William Brock, Cars Hommes and Florian Wagener, More hedging instruments may destabilize markets, WP06-11

15. Ginwestra Bianconi and Roberto Mulet, On the flexibility of complex systems, WP06-10

16. Ginwestra Bianconi and Matteo Marsili, Effect of degree correlations on the loop structure of scale-free networks, WP06-09

17. Ginwestra Bianconi, Tobias Galla and Matteo Marsili, Effects of Tobin Taxes in Minority Game Markets, WP06-08

18. Ginwestra Bianconi, Andrea De Martino, Felipe Ferreira and Matteo Marsili, Multi-asset minority games, WP06-07

19. Ba Chu, John Knight and Stephen Satchell, Optimal Investment and Asymmetric Risk for a Large Portfolio: A Large Deviations Approach, WP06-06

20. Ba Chu and Soosung Hwang, The Asymptotic Properties of AR(1) Process with the Occasionally Changing AR Coefficient, WP06-05

21. Ba Chu and Soosung Hwang, An Asymptotics of Stationary and Nonstationary AR(1) Processes with Multiple Structural Breaks in Mean, WP06-04

22. Ba Chu, Optimal Long Term Investment in a Jump Diffusion Setting: A Large Deviation Approach, WP06-03

23. Mikhail Anufriev and Gulio Bottazzi, Price and Wealth Dynamics in a Speculative Market with Generic Procedurally Rational Traders, WP06-02

24. Simonae Alfarano, Thomas Lux and Florian Wagner, Empirical Validation of Stochastic Models of Interacting Agents: A "Maximally Skewed" Noise Trader Model?, WP06-01

\section{5}

1. Shaun Bond and Soosung Hwang, Smoothing, Nonsynchronous Appraisal and CrossSectional Aggreagation in Real Estate Price Indices, WP05-17 
2. Mark Salmon, Gordon Gemmill and Soosung Hwang, Performance Measurement with Loss Aversion, WP05-16

3. Philippe Curty and Matteo Marsili, Phase coexistence in a forecasting game, WP05-15

4. Matthew Hurd, Mark Salmon and Christoph Schleicher, Using Copulas to Construct Bivariate Foreign Exchange Distributions with an Application to the Sterling Exchange Rate Index (Revised), WP05-14

5. Lucio Sarno, Daniel Thornton and Giorgio Valente, The Empirical Failure of the Expectations Hypothesis of the Term Structure of Bond Yields, WP05-13

6. Lucio Sarno, Ashoka Mody and Mark Taylor, A Cross-Country Financial Accelorator: Evidence from North America and Europe, WP05-12

7. Lucio Sarno, Towards a Solution to the Puzzles in Exchange Rate Economics: Where Do We Stand?, WP05-11

8. James Hodder and Jens Carsten Jackwerth, Incentive Contracts and Hedge Fund Management, WP05-10

9. James Hodder and Jens Carsten Jackwerth, Employee Stock Options: Much More Valuable Than You Thought, WP05-09

10. Gordon Gemmill, Soosung Hwang and Mark Salmon, Performance Measurement with Loss Aversion, WP05-08

11. George Constantinides, Jens Carsten Jackwerth and Stylianos Perrakis, Mispricing of S\&P 500 Index Options, WP05-07

12. Elisa Luciano and Wim Schoutens, A Multivariate Jump-Driven Financial Asset Model, WP0506

13. Cees Diks and Florian Wagener, Equivalence and bifurcations of finite order stochastic processes, WP05-05

14. Devraj Basu and Alexander Stremme, CAY Revisited: Can Optimal Scaling Resurrect the (C)CAPM?, WP05-04

15. Ginwestra Bianconi and Matteo Marsili, Emergence of large cliques in random scale-free networks, WP05-03

16. Simone Alfarano, Thomas Lux and Friedrich Wagner, Time-Variation of Higher Moments in a Financial Market with Heterogeneous Agents: An Analytical Approach, WP05-02

17. Abhay Abhayankar, Devraj Basu and Alexander Stremme, Portfolio Efficiency and Discount Factor Bounds with Conditioning Information: A Unified Approach, WP05-01

\section{4}

1. Xiaohong Chen, Yanqin Fan and Andrew Patton, Simple Tests for Models of Dependence Between Multiple Financial Time Series, with Applications to U.S. Equity Returns and Exchange Rates, WP04-19

2. Valentina Corradi and Walter Distaso, Testing for One-Factor Models versus Stochastic Volatility Models, WP04-18

3. Valentina Corradi and Walter Distaso, Estimating and Testing Sochastic Volatility Models using Realized Measures, WP04-17

4. Valentina Corradi and Norman Swanson, Predictive Density Accuracy Tests, WP04-16

5. Roel Oomen, Properties of Bias Corrected Realized Variance Under Alternative Sampling Schemes, WP04-15

6. Roel Oomen, Properties of Realized Variance for a Pure Jump Process: Calendar Time Sampling versus Business Time Sampling, WP04-14

7. Richard Clarida, Lucio Sarno, Mark Taylor and Giorgio Valente, The Role of Asymmetries and Regime Shifts in the Term Structure of Interest Rates, WP04-13

8. Lucio Sarno, Daniel Thornton and Giorgio Valente, Federal Funds Rate Prediction, WP04-12

9. Lucio Sarno and Giorgio Valente, Modeling and Forecasting Stock Returns: Exploiting the Futures Market, Regime Shifts and International Spillovers, WP04-11

10. Lucio Sarno and Giorgio Valente, Empirical Exchange Rate Models and Currency Risk: Some Evidence from Density Forecasts, WP04-10

11. Ilias Tsiakas, Periodic Stochastic Volatility and Fat Tails, WP04-09

12. Ilias Tsiakas, Is Seasonal Heteroscedasticity Real? An International Perspective, WP04-08

13. Damin Challet, Andrea De Martino, Matteo Marsili and Isaac Castillo, Minority games with finite score memory, WP04-07

14. Basel Awartani, Valentina Corradi and Walter Distaso, Testing and Modelling Market Microstructure Effects with an Application to the Dow Jones Industrial Average, WP04-06 
15. Andrew Patton and Allan Timmermann, Properties of Optimal Forecasts under Asymmetric Loss and Nonlinearity, WP04-05

16. Andrew Patton, Modelling Asymmetric Exchange Rate Dependence, WP04-04

17. Alessio Sancetta, Decoupling and Convergence to Independence with Applications to Functional Limit Theorems, WP04-03

18. Alessio Sancetta, Copula Based Monte Carlo Integration in Financial Problems, WP04-02

19. Abhay Abhayankar, Lucio Sarno and Giorgio Valente, Exchange Rates and Fundamentals: Evidence on the Economic Value of Predictability, WP04-01

\section{2}

1. Paolo Zaffaroni, Gaussian inference on Certain Long-Range Dependent Volatility Models, WP02-12

2. Paolo Zaffaroni, Aggregation and Memory of Models of Changing Volatility, WP02-11

3. Jerry Coakley, Ana-Maria Fuertes and Andrew Wood, Reinterpreting the Real Exchange Rate - Yield Diffential Nexus, WP02-10

4. Gordon Gemmill and Dylan Thomas, Noise Training, Costly Arbitrage and Asset Prices: evidence from closed-end funds, WP02-09

5. Gordon Gemmill, Testing Merton's Model for Credit Spreads on Zero-Coupon Bonds, WP0208

6. George Christodoulakis and Steve Satchell, On th Evolution of Global Style Factors in the MSCI Universe of Assets, WP02-07

7. George Christodoulakis, Sharp Style Analysis in the MSCI Sector Portfolios: A Monte Caro Integration Approach, WP02-06

8. George Christodoulakis, Generating Composite Volatility Forecasts with Random Factor Betas, WP02-05

9. Claudia Riveiro and Nick Webber, Valuing Path Dependent Options in the Variance-Gamma Model by Monte Carlo with a Gamma Bridge, WP02-04

10. Christian Pedersen and Soosung Hwang, On Empirical Risk Measurement with Asymmetric Returns Data, WP02-03

11. Roy Batchelor and Ismail Orgakcioglu, Event-related GARCH: the impact of stock dividends in Turkey, WP02-02

12. George Albanis and Roy Batchelor, Combining Heterogeneous Classifiers for Stock Selection, WP02-01

\section{1}

1. Soosung Hwang and Steve Satchell, GARCH Model with Cross-sectional Volatility; GARCHX Models, WP01-16

2. Soosung Hwang and Steve Satchell, Tracking Error: Ex-Ante versus Ex-Post Measures, WP01-15

3. Soosung Hwang and Steve Satchell, The Asset Allocation Decision in a Loss Aversion World, WP01-14

4. Soosung Hwang and Mark Salmon, An Analysis of Performance Measures Using Copulae, WP01-13

5. Soosung Hwang and Mark Salmon, A New Measure of Herding and Empirical Evidence, WP01-12

6. Richard Lewin and Steve Satchell, The Derivation of New Model of Equity Duration, WP0111

7. Massimiliano Marcellino and Mark Salmon, Robust Decision Theory and the Lucas Critique, WP01-10

8. Jerry Coakley, Ana-Maria Fuertes and Maria-Teresa Perez, Numerical Issues in Threshold Autoregressive Modelling of Time Series, WP01-09

9. Jerry Coakley, Ana-Maria Fuertes and Ron Smith, Small Sample Properties of Panel Timeseries Estimators with I(1) Errors, WP01-08

10. Jerry Coakley and Ana-Maria Fuertes, The Felsdtein-Horioka Puzzle is Not as Bad as You Think, WP01-07

11. Jerry Coakley and Ana-Maria Fuertes, Rethinking the Forward Premium Puzzle in a Nonlinear Framework, WP01-06

12. George Christodoulakis, Co-Volatility and Correlation Clustering: A Multivariate Correlated ARCH Framework, WP01-05 
13. Frank Critchley, Paul Marriott and Mark Salmon, On Preferred Point Geometry in Statistics, WP01-04

14. Eric Bouyé and Nicolas Gaussel and Mark Salmon, Investigating Dynamic Dependence Using Copulae, WP01-03

15. Eric Bouyé, Multivariate Extremes at Work for Portfolio Risk Measurement, WP01-02

16. Erick Bouyé, Vado Durrleman, Ashkan Nikeghbali, Gael Riboulet and Thierry Roncalli, Copulas: an Open Field for Risk Management, WP01-01

\section{0}

1. Soosung Hwang and Steve Satchell, Valuing Information Using Utility Functions, WP00-06

2. Soosung Hwang, Properties of Cross-sectional Volatility, WP00-05

3. Soosung Hwang and Steve Satchell, Calculating the Miss-specification in Beta from Using a Proxy for the Market Portfolio, WP00-04

4. Laun Middleton and Stephen Satchell, Deriving the APT when the Number of Factors is Unknown, WP00-03

5. George A. Christodoulakis and Steve Satchell, Evolving Systems of Financial Returns: AutoRegressive Conditional Beta, WP00-02

6. Christian S. Pedersen and Stephen Satchell, Evaluating the Performance of Nearest Neighbour Algorithms when Forecasting US Industry Returns, WP00-01

\section{9}

1. Yin-Wong Cheung, Menzie Chinn and Ian Marsh, How do UK-Based Foreign Exchange Dealers Think Their Market Operates?, WP99-21

2. Soosung Hwang, John Knight and Stephen Satchell, Forecasting Volatility using LINEX Loss Functions, WP99-20

3. Soosung Hwang and Steve Satchell, Improved Testing for the Efficiency of Asset Pricing Theories in Linear Factor Models, WP99-19

4. Soosung Hwang and Stephen Satchell, The Disappearance of Style in the US Equity Market, WP99-18

5. Soosung Hwang and Stephen Satchell, Modelling Emerging Market Risk Premia Using Higher Moments, WP99-17

6. Soosung Hwang and Stephen Satchell, Market Risk and the Concept of Fundamental Volatility: Measuring Volatility Across Asset and Derivative Markets and Testing for the Impact of Derivatives Markets on Financial Markets, WP99-16

7. Soosung Hwang, The Effects of Systematic Sampling and Temporal Aggregation on Discrete Time Long Memory Processes and their Finite Sample Properties, WP99-15

8. Ronald MacDonald and Ian Marsh, Currency Spillovers and Tri-Polarity: a Simultaneous Model of the US Dollar, German Mark and Japanese Yen, WP99-14

9. Robert Hillman, Forecasting Inflation with a Non-linear Output Gap Model, WP99-13

10. Robert Hillman and Mark Salmon, From Market Micro-structure to Macro Fundamentals: is there Predictability in the Dollar-Deutsche Mark Exchange Rate?, WP99-12

11. Renzo Avesani, Giampiero Gallo and Mark Salmon, On the Evolution of Credibility and Flexible Exchange Rate Target Zones, WP99-11

12. Paul Marriott and Mark Salmon, An Introduction to Differential Geometry in Econometrics, WP99-10

13. Mark Dixon, Anthony Ledford and Paul Marriott, Finite Sample Inference for Extreme Value Distributions, WP99-09

14. Ian Marsh and David Power, A Panel-Based Investigation into the Relationship Between Stock Prices and Dividends, WP99-08

15. Ian Marsh, An Analysis of the Performance of European Foreign Exchange Forecasters, WP99-07

16. Frank Critchley, Paul Marriott and Mark Salmon, An Elementary Account of Amari's Expected Geometry, WP99-06

17. Demos Tambakis and Anne-Sophie Van Royen, Bootstrap Predictability of Daily Exchange Rates in ARMA Models, WP99-05

18. Christopher Neely and Paul Weller, Technical Analysis and Central Bank Intervention, WP9904

19. Christopher Neely and Paul Weller, Predictability in International Asset Returns: A Reexamination, WP99-03 
20. Christopher Neely and Paul Weller, Intraday Technical Trading in the Foreign Exchange Market, WP99-02

21. Anthony Hall, Soosung Hwang and Stephen Satchell, Using Bayesian Variable Selection Methods to Choose Style Factors in Global Stock Return Models, WP99-01

\section{8}

1. Soosung Hwang and Stephen Satchell, Implied Volatility Forecasting: A Compaison of Different Procedures Including Fractionally Integrated Models with Applications to UK Equity Options, WP98-05

2. Roy Batchelor and David Peel, Rationality Testing under Asymmetric Loss, WP98-04

3. Roy Batchelor, Forecasting T-Bill Yields: Accuracy versus Profitability, WP98-03

4. Adam Kurpiel and Thierry Roncalli, Option Hedging with Stochastic Volatility, WP98-02

5. Adam Kurpiel and Thierry Roncalli, Hopscotch Methods for Two State Financial Models, WP98-01 